\title{
General theory of robustness against disorder in multi-band superconductors
}

\author{
D. C. Cavanagh ${ }^{1, *}$ and P. M. R. Brydon ${ }^{2, \dagger}$ \\ ${ }^{1}$ Department of Physics, University of Otago, P.O. Box 56, Dunedin 9054, New Zealand \\ ${ }^{2}$ Department of Physics and MacDiarmid Institute for Advanced Materials and Nanotechnology, \\ University of Otago, P.O. Box 56, Dunedin 9054, New Zealand
}

(Dated: 14 April, 2021)

\begin{abstract}
We investigate the influence of general forms of disorder on the robustness of superconductivity in multiband materials. Specifically, we consider a general two-band system where the bands arise from an orbital degree of freedom of the electrons. Within the Born approximation, we show that the interplay of the spin-orbital structure of the normal-state Hamiltonian, disorder scattering, and superconducting pairing potentials can lead to significant deviations from the expected robustness of the superconductivity. This can be conveniently formulated in terms of the so-called "superconducting fitness". In particular, we verify a key role for unconventional $s$-wave states, permitted by the spin-orbital structure and which may pair electrons that are not time-reversed partners. To exemplify the role of Fermi surface topology and spin-orbital texture, we apply our formalism to the candidate topological superconductor $\mathrm{Cu}_{x} \mathrm{Bi}_{2} \mathrm{Se}_{3}$, for which only a single band crosses the Fermi energy, as well as models of the iron pnictides, which possess multiple Fermi pockets.
\end{abstract}

\section{INTRODUCTION}

The influence of disorder on superconductivity is a significant, and frequently utilized, probe of the superconducting order parameter [1]. When pairing occurs between time-reversed partners, Anderson's theorem [2] states that disorder can depair electrons only when the superconducting gap is anisotropic or the disorder breaks time-reversal symmetry. The behavior of the critical temperature $T_{c}$ in the presence of disorder is, as a result, one of the key indicators of unconventional superconductivity in single band materials. Conventional (fully gapped) superconductivity is only sensitive to time-reversal symmetry breaking (TRSB), or magnetic, disorder, while unconventional superconductors are equally susceptible to both time-reversal symmetric (TRS) disorder and TRSB disorder.

In many superconductors of recent interest, the lowenergy electronic states are conveniently labelled by discrete quantum numbers additional to spin, e.g. the atomic orbital or sublattice site from each the electrons originate. The existence of these novel "orbital" degree of freedom has been proposed to play an important role in both the normal state and superconducting properties [3 8. In such superconductors, pairing may be isotropic in momentum without pairing time-reversed states [9[1], and there has hence been much recent interest in generalizing Anderson's theorem to account for such systems [18, 25]. The effect of disorder in such systems is considerably more complicated due to the interplay of the internal spin-orbital structure of the superconducting states with the spin-orbital texture of the electronic system.

Previously, we developed a framework to illustrate the significant role played by the spin-orbital texture

* david.cavanagh@otago.ac.nz

$\dagger$ philip.brydon@otago.ac.nz in determining the robustness of various superconducting states, even those with momentum-dependent pairing functions, against scalar TRS disorder with no dependence on the internal degrees of freedom [19. In this work, we extend this framework to consider both TRS and TRSB disorder with a non-trivial dependence on the internal degrees of freedom. Additionally, we highlight the increased robustness of anisotropic pairing states due to the existence of the unconventional $s$-wave states, and demonstrate how the number of bands at the Fermi level influences the robustness.

As a demonstration of the utility of our framework, we apply it to two families of materials proposed to realize unconventional $s$-wave states: superconducting Dirac systems (such as the apparently nematic and fullygapped superconductor $\mathrm{Cu}_{x} \mathrm{Bi}_{2} \mathrm{Se}_{3}$ [9, 26, 30]) and the iron pnictide superconductors [10, 31. The orbital degree of freedom has a significant influence on the superconductivity in the Dirac materials, though only a single band crosses the Fermi level [9, 18, 23, 26, 30. We demonstrate, for $\mathrm{Cu}_{x} \mathrm{Bi}_{2} \mathrm{Se}_{3}$, the key role played by the spin-orbital structure of the disorder potential in determining the robustness of various superconducting states.

In the iron-based pnictide superconductors, multiple bands cross the Fermi level leading to a Fermi surface with multiple sheets. The consensus view of experiment and theory is that an $s^{ \pm}$-wave state is realized in the majority of these materials, where each Fermi sheet has a largely isotropic gap but with opposite sign between the electron- and hole-like Fermi surfaces 32 35. There is an extensive literature on the effect of impurities on this pairing state, see e.g. 36 42. Nevertheless, a variety of more exotic superconducting states have been proposed that exploit the striking orbital texture of the Fermi surfaces [10, 16, 43. Motivated by these works, and to emphasize the important role of Fermi surface spin-orbital polarization and topology, we examine a number of superconducting states which are possible in a well-known two-orbital model [4] for these materials. 
Our paper is organized as follows: In Sec. II] we develop the generalization of our theoretical framework, based on the self-consistent Born approximation, to account for the influence of non-scalar disorder on superconductivity in two-band systems. In Sec. III, we apply this framework to models of superconducting Dirac materials and highlight that the time-reversal symmetry, or lack thereof, of the disorder scattering is not the dominant factor responsible for determining the robustness of a given superconducting state. We consider, in Sec. IV] the additional influence of multiple Fermi surface pockets in more detail by applying our framework to the iron pnictide superconductors. In Sec. V] we discuss some general insights, in particular the important role played by the superconducting fitness [6, 19, 45, and make reconcile discrepancies between some recent results and our own.

\section{THEORY}

Our general framework is developed for a generic twoband system with both inversion and time-reversal symmetry. States in such a system are typically defined by the electron spin and a quantum number associated with electron orbital, sublattice or some other additional degree of freedom. In general, we refer to the four degrees of freedom as a 'spin-orbital' basis, regardless of the origin of the additional degree of freedom.

\section{A. Normal state properties}

We consider systems with the normal-state Hamiltonian $H=\sum_{\boldsymbol{k}} c_{\boldsymbol{k}}^{\dagger} \mathcal{H}_{\mathbf{k}} c_{\boldsymbol{k}}$, where $c_{\boldsymbol{k}}$ is a four component spinor encoding the internal degrees of freedom. The most general form of the matrix $\mathcal{H}_{\boldsymbol{k}}$ is [4],

$$
\mathcal{H}_{\boldsymbol{k}}=\epsilon_{\boldsymbol{k}, 0} \mathbb{1}_{4}+\vec{\epsilon}_{\boldsymbol{k}} \cdot \vec{\gamma}
$$

where $\mathbb{1}_{4}$ is the $4 \times 4$ unit matrix and $\vec{\gamma}=$ $\left(\gamma^{1}, \gamma^{2}, \gamma^{3}, \gamma^{4}, \gamma^{5}\right)$ are the five mutually anti-commuting Euclidean Dirac matrices. The coefficients of these matrices $\epsilon_{\boldsymbol{k}, 0}$ and $\vec{\epsilon}_{\boldsymbol{k}}=\left(\epsilon_{\boldsymbol{k}, 1}, \epsilon_{\boldsymbol{k}, 2}, \epsilon_{\boldsymbol{k}, 3}, \epsilon_{\boldsymbol{k}, 4}, \epsilon_{\boldsymbol{k}, 5}\right)$ are all real functions. The eigenvalues of Eq. (1) are the band energies

$$
E_{\boldsymbol{k}, \pm}=\epsilon_{\boldsymbol{k}, 0} \pm\left|\vec{\epsilon}_{\boldsymbol{k}}\right|
$$

The anticommutation of the $\gamma$ matrices among themselves ensures that these eigenvalues are doubly degenerate, reflecting the presence of inversion and timereversal symmetry. Time-reversal is given by the operator $\mathcal{T}=U_{T} \mathcal{K}$, where $\mathcal{K}$ is complex conjugation and the unitary part of the time-reversal operator can be chosen as $U_{T}=\gamma^{3} \gamma^{5}$ without loss of generality. Inversion symmetry either affects the internal degrees of freedom trivially $\left(\mathcal{I}=\mathbb{1}_{4}\right)$ or nontrivially $\left(\mathcal{I}=\gamma^{1}\right)$. Due to the presence of time-reversal and inversion symmetry, it is generally possible to label the two-fold-degenerate eigenstates of the Hamiltonian in terms of a pseudospin index, which behaves like a spin- $\frac{1}{2}$ under these two symmetries. Several authors have analyzed the impurity problem in terms a band-pseudospin or similar basis [25, 30]. Although this has the advantage of casting the pairing potentials in a more familiar form, we do not pursue this approach as it obscures the important role of the orbitalspin degree of freedom.

We consider isotropic scattering off potential impurities of different types $\alpha$ distributed randomly at positions $\boldsymbol{r}_{j_{\alpha}}$, described by the Hamiltonian

$$
H_{\mathrm{imp}}=\frac{1}{\Omega} \sum_{\alpha} \sum_{j_{\alpha}} \sum_{\boldsymbol{k}, \boldsymbol{k}^{\prime}} e^{i\left(\boldsymbol{k}^{\prime}-\boldsymbol{k}\right) \cdot \boldsymbol{r}_{j_{\alpha}}} c_{\boldsymbol{k}}^{\dagger} \tilde{V}_{\alpha} c_{\boldsymbol{k}^{\prime}}
$$

where $\Omega$ is the volume and $\tilde{V}_{\alpha}=V_{\alpha} \gamma^{\alpha_{x}} \gamma^{\alpha_{y}}$ is the impurity potential. The choice $\alpha_{x}=\alpha_{y}=0$ corresponds to the scalar disorder considered in Ref. [19. Note that our theory allows both for distinct impurities of different types (i.e. $\mathbf{r}_{j_{\alpha}} \neq \mathbf{r}_{j_{\tilde{\alpha}}}$ for $\alpha \neq \tilde{\alpha}$ ), or for the impurities to have multiple different scattering potentials (i.e. $\mathbf{r}_{j_{\alpha}}=\mathbf{r}_{j_{\tilde{\alpha}}}$ ). Within the Born approximation, the effect of the impurities is accounted for via a self-energy $\Sigma_{1}\left(i \omega_{n}\right)$, so that the full Green's function satisfies the Dyson equation

$$
\bar{G}^{-1}\left(\boldsymbol{k}, i \omega_{n}\right)=G_{0}^{-1}\left(\boldsymbol{k}, i \omega_{n}\right)-\Sigma_{1}\left(i \omega_{n}\right)
$$

where $G_{0}\left(\boldsymbol{k}, i \omega_{n}\right)$ is the Green's function of the clean system and the self-energy is determined self-consistently

$$
\Sigma_{1}\left(i \omega_{n}\right)=\sum_{\alpha} n_{\mathrm{imp}, \alpha} \tilde{V}_{\alpha} \int \frac{d^{3} k}{(2 \pi)^{3}} \bar{G}\left(\boldsymbol{k}, i \omega_{n}\right) \tilde{V}_{\alpha}^{\dagger},
$$

where $n_{\mathrm{imp}, \alpha}$ is the concentration of $\alpha$-type impurities. This approximation is valid when disorder scattering is weak relative to the chemical potential, $\hbar \tau^{-1} \ll \mu$, where $\tau^{-1}$ is the disorder scattering rate. If we further require that the disorder scattering is small compared to the band separation $\left|\vec{\epsilon}_{\boldsymbol{k}}\right|$ at the Fermi surface, then to leading order in $\hbar \tau^{-1} /\left|\vec{\epsilon}_{\boldsymbol{k}}\right|$ the Green's functions of the disordered system is

$$
\bar{G}\left(\boldsymbol{k}, i \omega_{n}\right)=\sum_{j= \pm} \frac{1}{i \tilde{\omega}_{n, j}-E_{\boldsymbol{k}, j}} \mathcal{P}_{\boldsymbol{k}, j}
$$

where $\mathcal{P}_{\boldsymbol{k}, \pm}=\frac{1}{2}\left(\mathbb{1}_{4} \pm \hat{\epsilon}_{\boldsymbol{k}} \cdot \vec{\gamma}\right)$ projects into the \pm band at momentum $\boldsymbol{k}$ and $\hat{\epsilon}_{\boldsymbol{k}}=\vec{\epsilon}_{\boldsymbol{k}} /\left|\vec{\epsilon}_{\boldsymbol{k}}\right|$. The effect of impurities on the normal state is accounted for by the renormalized Matsubara frequencies

$$
\tilde{\omega}_{n, j}=\omega_{n}-\frac{1}{2 \tau_{\boldsymbol{k}, j}} \operatorname{sgn}\left(\omega_{n}\right),
$$

where the scattering rate (SR) in band $j$ is given by

$$
\frac{1}{\tau_{\boldsymbol{k}, j}}=\sum_{\alpha} \pi n_{\mathrm{imp}, \alpha}\left|V_{\alpha}\right|^{2}
$$




$$
\times \sum_{m= \pm} \mathcal{N}_{m}\left(1+j m \sum_{i=1}^{5} \phi_{\alpha}^{(i)} \hat{\epsilon}_{\boldsymbol{k}, i}\left\langle\hat{\epsilon}_{\boldsymbol{k}, i}\right\rangle_{m}\right)
$$

with $\mathcal{N}_{m}$ the density of states of band $m= \pm$ at the Fermi surface, and $\langle\ldots\rangle_{m}$ denotes the average over the Fermi surface of this band. The second term in the parentheses of Eq. (8) arises from a net average polarization in the internal degrees of freedom on the Fermi surface of the $m$ th band 19. The factor $\phi_{\alpha}^{(i)}=+1(-1)$ if the scattering potential $\tilde{V}_{\alpha}$ commutes (anticommutes) with the term $\epsilon_{\boldsymbol{k}, i} \gamma^{i}$ in the normal-state Hamiltonian Eq. (1). This contribution can have important effects on the overall scattering rate, enhancing or reducing the relative magnitude of interband to intraband scattering. In the following we will assume a weak momentum-dependence of the SR and replace $\tau_{\mathbf{k}, j}^{-1}$ by its Fermi surface average $\tau_{j}^{-1}$ in Eq. 6 .

\section{B. Superconducting properties}

In the orbital-spin basis, the pairing potential for a general superconducting state is $\Delta_{k}=\Delta_{0} \tilde{\Delta}_{k}$ where $\Delta_{0}$ is the magnitude and

$$
\tilde{\Delta}_{\boldsymbol{k}}=f_{\boldsymbol{k}} \gamma^{\alpha} \gamma^{\beta} U_{T}
$$

Here $f_{\boldsymbol{k}}$ is a normalized form factor, which must be chosen such that fermionic antisymmetry is satisfied, i.e. $\tilde{\Delta}_{\boldsymbol{k}}=-\tilde{\Delta}_{-\boldsymbol{k}}^{T}$. The pairing potential $\tilde{\Delta}_{\boldsymbol{k}}$ is a $4 \times 4$ matrix, and so there are only six matrices defined by Eq. (9) for which an even-parity form factor (i.e. $f_{\boldsymbol{k}}=f_{-\boldsymbol{k}}$ ) is permitted, while the other ten matrices must have an odd-parity form factor (i.e. $f_{\boldsymbol{k}}=-f_{-\boldsymbol{k}}$ ).

The pairing states which have $s$-wave form factor $\left(f_{\boldsymbol{k}}=\right.$ 1) are of central importance to our theory. One such state is always given by $\alpha=\beta=0$ (where $\gamma^{0}=\mathbb{1}_{4}$ ), which describes the pairing of electrons in time-reversed-partner states, and is hence the generalization of the single-band conventional $s$-wave spin-singlet state. The remaining five $s$-wave channels depend nontrivially on the orbital degrees of freedom, i.e. $\alpha \neq \beta$. The pairing potentials are determined by the form of the inversion operator: for a trivial inversion operator we have

$$
(\alpha, \beta)=(0,1),(0,2),(0,3),(0,4),(0,5)
$$

whereas for a nontrivial inversion operator $\mathcal{I}=\gamma^{1}$ the five potentials are

$$
(\alpha, \beta)=(0,1),(1,2),(1,3),(1,4),(1,5) .
$$

These additional $s$-wave channels may belong to nontrivial irreps, and in general involve both intraband and interband pairing. When projected onto the Fermi surface, these states will typically have non-trivial momentumdependence and gap nodes may be present [19, 23].

The degree to which the $s$-wave states involve interband pairing can be quantified by the "superconducting fitness" 6, 45]. For convenience, we define the normalized superconducting fitness on the $j$ th band

$$
\tilde{F}_{C}^{(j)}=\left\langle\frac{\operatorname{Tr}\left\{\left|\mathcal{H}_{\boldsymbol{k}} \tilde{\Delta}_{\boldsymbol{k}}-\tilde{\Delta}_{\boldsymbol{k}} \mathcal{H}_{-\boldsymbol{k}}^{T}\right|^{2}\right\}}{\left|\vec{\epsilon}_{\boldsymbol{k}}\right|^{2} \operatorname{Tr}\left\{\tilde{\Delta}_{\boldsymbol{k}} \tilde{\Delta}_{\boldsymbol{k}}^{\dagger}\right\}}\right\rangle_{j} .
$$

By definition, we have $0 \leq \tilde{F}_{C}^{(j)} \leq 1$; this quantity is related to the magnitude of the gap in the quasiparticle dispersion on the Fermi surface $j$ by

$$
\left|\Delta_{\mathbf{k}, j}\right|=\Delta_{0} \sqrt{1-\tilde{F}_{C}^{(j)}} .
$$

When the pairing is purely intraband, the fitness $\tilde{F}_{C}^{(j)}$ is vanishing and the gap takes a maximal value. Conversely, we see that gap nodes correspond to lines or points on the Fermi surface where the gap is maximally unfit, i.e. the pairing is purely interband.

The fitness for the conventional $s$-wave state is equal to zero, consistent with Anderson's theorem. The fitness for the unconventional $s$-wave states in a system with trivial inversion symmetry Eq. 10 evaluates as

$$
\tilde{F}_{C}^{(j)}=1-\left\langle\hat{\epsilon}_{\boldsymbol{k}, \beta}^{2}\right\rangle_{j}
$$

whereas in the system with nontrivial inversion Eq. 11 the fitness for the unconventional $s$-wave states is

$$
\tilde{F}_{C}^{(j)}= \begin{cases}1-\left\langle\hat{\epsilon}_{\boldsymbol{k}, 1}^{2}\right\rangle_{j} & (\alpha, \beta)=(0,1) \\ \left\langle\hat{\epsilon}_{\boldsymbol{k}, 1}^{2}+\hat{\epsilon}_{\boldsymbol{k}, n}^{2}\right\rangle_{j} & (\alpha, \beta)=(1, n)\end{cases}
$$

Note that the odd-parity $s$-wave states typically have smaller values of $\tilde{F}_{C}^{(j)}$ than the even-parity $s$-wave states.

Expressed in a band-pseudospin basis, a pairing potential will typically have both inter- and intraband components. In particular, the intraband components will either be pseudospin-singlet or triplet, according as it is even or odd parity. Although the intraband pairing potential in the latter case is dependent upon the pseudospin basis, projecting the former into the pseudospin basis we find

$$
\Delta_{0} \gamma^{\beta} U_{T} \rightarrow_{ \pm} \begin{cases}\Delta_{0} i s_{y} & \beta=0 \\ \pm \Delta_{0} \hat{\epsilon}_{\mathbf{k}, \beta} i s_{y} & \beta \neq 0\end{cases}
$$

where $s_{y}$ is the Pauli matrix in the pseudospin degree of freedom. In particular, we note that for the unconventional $(\beta \neq 0)$ states the sign of the intraband potential reverses between the two bands.

\section{The anomalous self-energy}

We now apply the Born approximation to the superconducting state, specifically to determine the effect of the disorder on the critical temperature. We distinguish between two cases: where pairing occurs in only a single channel, and where multiple distinct pairing channels are present. 


\section{Single channel}

The pairing potential is determined self-consistently from the equation

$$
\Delta_{0}=\frac{g_{\nu}}{2 \beta} \sum_{i \omega_{n}} \int \frac{d^{3} k}{(2 \pi)^{3}} \operatorname{Tr}\left\{\tilde{\Delta}_{\boldsymbol{k}}^{\dagger} \bar{F}\left(\mathbf{k}, i \omega_{n}\right)\right\}
$$

where $g_{\nu}<0$ is the attractive interaction in a particular superconducting channel $\nu$ and $\bar{F}\left(\mathbf{k}, i \omega_{n}\right)$ is the impurityaveraged anomalous Green's function. In order to determine the critical temperature, we expand the anomalous Green's function to linear order in the pairing potential,

$$
\bar{F}\left(\mathbf{k}, i \omega_{n}\right) \approx \bar{G}\left(\boldsymbol{k}, i \omega_{n}\right)\left(\Delta_{\boldsymbol{k}}+\Sigma_{2}\right) \bar{G}_{h}\left(\boldsymbol{k}, i \omega_{n}\right)
$$

where $\bar{G}_{h}\left(\boldsymbol{k}, i \omega_{n}\right)=\bar{G}^{T}\left(-\boldsymbol{k}, i \omega_{n}\right)$ is the impurityaveraged normal-state Green's function for the holes and $\Sigma_{2}$ is the anomalous self-energy due to the impurity scattering. Inserting this into Eq. 17] we obtain the linearized gap equation

$$
\begin{aligned}
\Delta_{0}= & \frac{g_{\nu}}{2 \beta} \sum_{i \omega_{n}} \int \frac{d^{3} k}{(2 \pi)^{3}} \operatorname{Tr}\left\{\tilde{\Delta}_{\boldsymbol{k}}^{\dagger} \bar{G}\left(\boldsymbol{k}, i \omega_{n}\right)\right. \\
& \left.\times\left(\Delta_{\boldsymbol{k}}+\Sigma_{2}\right) \bar{G}_{h}\left(\boldsymbol{k}, i \omega_{n}\right)\right\}
\end{aligned}
$$

The influence of disorder on the superconductivity is captured by the anomalous self-energy, which obeys the selfconsistency condition

$$
\begin{aligned}
\Sigma_{2}= & -\sum_{\alpha} n_{\mathrm{imp}, \alpha} \tilde{V}_{\alpha} \int \frac{d^{3} k}{(2 \pi)^{3}} \bar{G}\left(\boldsymbol{k}, i \omega_{n}\right) \\
& \times\left(\Delta_{\boldsymbol{k}}+\Sigma_{2}\right) \bar{G}_{h}\left(\boldsymbol{k}, i \omega_{n}\right) \tilde{V}_{\alpha}^{T} .
\end{aligned}
$$

Importantly, the anomalous self-energy vanishes unless the lowest-order contribution is nonzero:

$$
\begin{aligned}
\Sigma_{2}^{(0)} & =-\sum_{\alpha} n_{\mathrm{imp}, \alpha} \tilde{V}_{\alpha} \int \frac{d^{3} k}{(2 \pi)^{3}} \bar{G}\left(\boldsymbol{k}, i \omega_{n}\right) \Delta_{\boldsymbol{k}} \bar{G}_{h}\left(\boldsymbol{k}, i \omega_{n}\right) \tilde{V}_{\alpha}^{T} \\
& =\pi \sum_{\alpha} n_{\mathrm{imp}, \alpha} \tilde{V}_{\alpha} \sum_{j= \pm} \frac{\mathcal{N}_{j}}{\left|\tilde{\omega}_{n, j}\right|}\left\langle\mathcal{P}_{\boldsymbol{k}, j} \Delta_{\boldsymbol{k}} \mathcal{P}_{-\boldsymbol{k}, j}^{T}\right\rangle_{j} \tilde{V}_{\alpha}^{T}
\end{aligned}
$$

In the final line of Eq. 21, we have neglected contributions to the self-energy due to interband pairing, on the the assumption that the energy separation of the bands is much larger than the characteristic energy scales of the superconductivity and the impurity scattering.

For the two-band system considered here, the lowestorder contribution to the anomalous self-energy for the general pairing state Eq. (9) is

$\Sigma_{2}^{(0)}=\sum_{\alpha} \pi n_{\mathrm{imp}, \alpha} \tilde{V}_{\alpha} \sum_{j= \pm} \frac{\mathcal{N}_{j}}{4\left|\tilde{\omega}_{n, j}\right|} \Delta_{0}\left[\left\langle f_{\boldsymbol{k}}\right\rangle_{j} \gamma^{\alpha} \gamma^{\beta}+j \sum_{l=1}^{5}\left\langle f_{\boldsymbol{k}} \hat{\epsilon}_{\boldsymbol{k}, l}\right\rangle_{j}\left\{\gamma^{\alpha} \gamma^{\beta}, \gamma^{l}\right\}+\sum_{l, m=1}^{5}\left\langle f_{\boldsymbol{k}} \hat{\epsilon}_{\boldsymbol{k}, l} \hat{\epsilon}_{\boldsymbol{k}, m}\right\rangle_{j} \gamma^{l} \gamma^{\alpha} \gamma^{\beta} \gamma^{m}\right] U_{T} \tilde{V}_{\alpha}^{T}$

Since $\Sigma_{2}^{(0)}$ is an anomalous self-energy, is must satisfy the fermionic antisymmetry condition

$$
\Sigma_{2}^{(0)}\left(i \omega_{n}\right)=-\Sigma_{2}^{(0) T}\left(-i \omega_{n}\right)
$$

As seen from 22, the self-energy is even in frequency and thus the only non-vanishing terms allowed in $\Sigma_{2}^{(0)}$ (and therefore also $\Sigma_{2}$ ) are those proportional to the unconventional $s$-wave potentials [19].

As was the case for the normal scattering rate, accounting for the non-trivial structure of the disorder potentials necessitates the inclusion of an additional parameter, $\lambda_{\alpha}=+1(-1)$ if the gap is fit (unfit) with respect to the scattering potential, i.e. $\tilde{V}_{\alpha} \tilde{\Delta}_{k}-\tilde{\Delta}_{k} \tilde{V}_{\alpha}^{T}=0(\neq 0)$. For a conventional $s$-wave gap, $\tilde{\Delta}=U_{T}$, this condition is exactly equivalent to whether the disorder potential preserves or breaks time-reversal symmetry. In analogy with the conventional case, for unconventional $s$-wave states we will refer to scattering potentials as 'nonmagnetic' or 'magnetic' according as they are fit or unfit with respect to the pairing potential.

The lowest-order contribution to the anomalous self- energy for the $s$-wave state $\nu$ can be expressed as

$$
\Sigma_{2}^{(0)}=\sum_{\alpha} \pi n_{\mathrm{imp}, \alpha}\left|V_{\alpha}\right|^{2} \lambda_{\alpha} \sigma_{2,0} \tilde{\Delta}_{\nu}
$$

where the form of $\sigma_{2,0}$ depends on the fitness functions

$$
\sigma_{2,0}=\frac{1}{2}\left[\mathcal{N}_{+} \frac{1-\tilde{F}_{C}^{(+)}}{\left|\omega_{n}\right|+\tau_{+}^{-1}}+\mathcal{N}_{-} \frac{1-\tilde{F}_{C}^{(-)}}{\left|\omega_{n}\right|+\tau_{-}^{-1}}\right] .
$$

In particular, we observe that $\Sigma_{2}^{(0)}$ is only vanishing if the fitness functions evaluate to 1 , i.e. the state corresponds to purely interband pairing. Since such a situation is not thermodynamically stable in the weakcoupling regime [45, it will be generally true that an unconventional $s$-wave state with a nonzero critical temperature has a nonzero anomalous self-energy.

\section{Multiple channels}

We consider a general pairing state

$$
\Delta_{k}=\sum_{\mu} \Delta_{0}^{(\mu)} \tilde{\Delta}_{\mu, k}
$$


where each channel $\mu$ belongs to the same irrep. The pairing amplitudes $\Delta_{0}^{(\mu)}$ are fixed by solving the selfconsistency equations

$$
\Delta_{0}^{(\mu)}=\sum_{\nu} \frac{g_{\mu, \nu}}{2 \beta} \sum_{i \omega_{n}} \int \frac{d^{3} k}{(2 \pi)^{3}} \operatorname{Tr}\left\{\tilde{\Delta}_{\nu, \mathbf{k}}^{\dagger} \bar{F}\left(\mathbf{k}, i \omega_{n}\right)\right\}
$$

where $g_{\mu, \nu}$ is the pairing interaction which scatters a Cooper pair in channel $\mu$ into channel $\nu$. To determine the critical temperature we again linearize the anomalous
Green's function to obtain the linearized gap equation

$$
\begin{aligned}
\Delta_{0}^{(\mu)}= & \sum_{\nu} \frac{g_{\mu, \nu}}{2 \beta} \sum_{i \omega_{n}} \int \frac{d^{3} k}{(2 \pi)^{3}} \operatorname{Tr}\left\{\tilde{\Delta}_{\nu, \boldsymbol{k}}^{\dagger} \bar{G}\left(\boldsymbol{k}, i \omega_{n}\right)\right. \\
& \left.\times\left(\Delta_{\boldsymbol{k}}+\Sigma_{2}\right) \bar{G}_{h}\left(\boldsymbol{k}, i \omega_{n}\right)\right\}
\end{aligned}
$$

where we have introduced the anomalous self-energy $\Sigma_{2}$ which is determined self-consistently according to Eq. (20).

The anomalous self-energy will generally couple the various superconducting channels to the $s$-wave states in the same irrep; we see this explicitly in the lowest-order contribution

$$
\Sigma_{2}^{(0)}=\pi \sum_{\mu} \sum_{\alpha} n_{\mathrm{imp}, \alpha} \tilde{V}_{\alpha} \sum_{j= \pm} \frac{\mathcal{N}_{j}}{\left|\tilde{\omega}_{n, j}\right|}\left\langle\mathcal{P}_{\boldsymbol{k}, j} \Delta_{0}^{(\mu)} \tilde{\Delta}_{\mu} \mathcal{P}_{-\boldsymbol{k}, j}^{T}\right\rangle_{j} \tilde{V}_{\alpha}^{T}=\sum_{\mu} \sum_{\nu \in s \text {-wave }} \Delta_{0}^{(\mu)} \sigma_{2,0}^{(\mu, \nu)} \tilde{\Delta}_{\nu}
$$

where the indice $\mu(\nu)$ runs over the components of the order parameter in all the (only the $s$-wave) channels. The contribution to the self-energy in the $\nu s$-wave channel due to the gap in the $\mu$ channel is explicitly

$$
\begin{aligned}
\sigma_{2,0}^{(\mu, \nu)}= & \sum_{\alpha} \pi n_{\text {imp }, \alpha}\left|V_{\alpha}\right|^{2} \lambda_{\alpha}^{(\nu)} \\
& \sum_{j= \pm} \frac{\mathcal{N}_{j}}{4\left|\tilde{\omega}_{n, j}\right|} \operatorname{Tr}\left\{\left(\left\langle\mathcal{P}_{\boldsymbol{k}, j} \tilde{\Delta}_{\mu, \mathbf{k}} \mathcal{P}_{-\boldsymbol{k}, j}^{T}\right\rangle_{j}\right) \tilde{\Delta}_{\nu}^{\dagger}\right\} .
\end{aligned}
$$

where $\lambda_{\alpha}^{(\nu)}=+1(-1)$ has the same meaning as in the single channel case, for each individual channel $\nu$. The trace in this expression can be understood as a measure of the overlap of the pairing state $\mu$ with the $s$-wave channel $\nu$ on the Fermi surface $j$. The full anomalous self-energy is

$$
\Sigma_{2}=\sum_{\mu, \nu} \Delta_{0}^{(\mu)} \sigma_{2}^{(\mu, \nu)} \tilde{\Delta}_{\nu}
$$

with $\sigma_{2}$ given by the matrix equation

$$
\sigma_{2}=\left(\mathbb{1}-\sigma_{2,0}\right)^{-1} \sigma_{2,0},
$$

where $\sigma_{2,0}$ has matrix elements $\sigma_{2,0}^{(\mu, \nu)}$, which we take to be zero if $\nu$ does not correspond to an $s$-wave channel. Inserting the self-energy into the linearized gap equation (28), the critical temperature is determined by the solution $\operatorname{det}\{\mathcal{M}\}=0$, with

$$
\mathcal{M}_{i, j}=\delta_{i, j}-\sum_{\nu} \frac{g_{i, \nu}}{2 \beta} \sum_{i \omega_{n}} \int \frac{d^{3} k}{(2 \pi)^{3}} \operatorname{Tr}\left\{\tilde{\Delta}_{\nu, \boldsymbol{k}}^{\dagger} \bar{G}\left(\boldsymbol{k}, i \omega_{n}\right)\left[\left(\mathbb{1}-\sigma_{2,0}\right)^{-1}\right]^{(i, j)} \tilde{\Delta}_{j, \boldsymbol{k}} \bar{G}_{h}\left(\boldsymbol{k}, i \omega_{n}\right)\right\}
$$

Note that in the following we will only consider the case where the pairing interaction is diagonal, i.e. $g_{\nu, \mu}=$ $g_{\nu} \delta_{\nu, \mu}$.

\section{APPLICATION TO DIRAC SYSTEMS}

We first consider the application of our formalism to a Dirac-like systems, as an example of the case where only a single band crosses the Fermi energy. For concreteness, we focus on the potential topological super- conductor $\mathrm{Cu}_{x} \mathrm{Bi}_{2} \mathrm{Se}_{3}$, making contact with previous work [18, 19, 23, 25].

The low-energy electronic states in $\mathrm{Bi}_{2} \mathrm{Se}_{3}$ originate from the outermost Se sites of the $\mathrm{Bi}_{2} \mathrm{Se}_{3}$ quintuple layers. These Se sites are interchanged by inversion, and so give rise to a sublattice structure. To lowest order in $\boldsymbol{k}$ in each coefficient in Eq.11, the Hamiltonian is given by [47]

$$
\begin{aligned}
H= & -\mu \sigma_{0} \otimes \eta_{0}+m \sigma_{0} \otimes \eta_{x}+v_{z} k_{z} \sigma_{0} \otimes \eta_{y} \\
& +v\left(k_{x} \sigma_{y}-k_{y} \sigma_{x}\right) \otimes \eta_{z}+\lambda k_{x}\left(k_{x}^{2}-3 k_{y}^{2}\right) \sigma_{z} \otimes \eta_{z}
\end{aligned}
$$


where $\sigma_{\nu}\left(\eta_{\nu}\right)$ are Pauli matrices in spin (sublattice) space. The $\gamma$ matrices are defined $\vec{\gamma}=\left(\sigma_{0} \otimes \eta_{x}, \sigma_{0} \otimes\right.$ $\left.\eta_{y}, \sigma_{x} \otimes \eta_{z}, \sigma_{y} \otimes \eta_{z}, \sigma_{z} \otimes \eta_{z}\right)$. The inversion symmetry operator is $\mathcal{I}=\sigma_{0} \otimes \eta_{x}$; this term also appears Hamiltonian as the mass term $m \sigma_{0} \otimes \eta_{x}$, which gaps out the Dirac point at the Brillouin zone centre. The copper intercalation only very weakly alters the bandstructure of the topological insulator $\mathrm{Bi}_{2} \mathrm{Se}_{3}$, but dopes electrons into the system, so that only the upper band crosses the Fermi energy. For this system, there are four unconventional $s$-wave states belonging to odd-parity irreps of the $D_{3 h}$ point group, and an additional unconventional $s$-wave state belonging to the trivial $A_{1 g}$ representation 9 . We tabulate the relevant signs of $\lambda_{\alpha}$ for the $s$-wave states for all possible impurity potentials in Table $\mathrm{I}$.

\section{A. Odd-parity states}

Solving the linearized gap equation Eq. 19, the critical temperature of the odd-parity $s$-wave pairing states in the presence of disorder is given by the solution of

$$
\log \left(\frac{T_{c}}{T_{c 0}}\right)=\psi\left(\frac{1}{2}\right)-\psi\left(\frac{1}{2}+\frac{1}{4 \pi k_{B} T_{c} \tilde{\tau}_{\nu}}\right)
$$

with the effective scattering rate is given by

$$
\tilde{\tau}_{\nu}^{-1}=\tau^{-1}-\sum_{\alpha} \pi \lambda_{\alpha} n_{\mathrm{imp}, \alpha}\left|V_{\alpha}\right|^{2} \mathcal{N}\left(1-\tilde{F}_{C}\right)
$$

and the normal-state scattering rate is

$$
\tau^{-1}=\sum_{\alpha} \pi n_{\mathrm{imp}, \alpha}\left|V_{\alpha}\right|^{2} \mathcal{N}\left(1+\phi_{\alpha}\langle\hat{m}\rangle^{2}\right)
$$

Note that the mass term $m \sigma_{0} \otimes \eta_{x}$ generates a nonvanishing net spin-orbital polarization of the states at the Fermi surface 18, 19. Since only the + band crosses the Fermi energy, we drop the + subscript on the density of states in these formulas.

As can be readily seen in Table I] for each $s$-wave state there exists six potentials (always including scalar disor$\operatorname{der} \tilde{V}_{\alpha}=\mathbb{1}_{4}$ ) for which $\lambda_{\alpha}=+1$ ("non-magnetic"), and the other ten potentials have $\lambda_{\alpha}=-1$ ("magnetic"). If the disorder potential has $\lambda_{\alpha}=+1$, the effective SR is reduced by an amount proportional to the degree of fitness of the pairing potential: the fitter the gap (and therefore the smaller $\tilde{F}_{C}$ ), the larger the reduction in the scattering rate. In contrast, when $\lambda_{\alpha}=-1$ the $\mathrm{SR}$ is instead enhanced, and the enhancement increases with increasing fitness. Even if only "nonmagnetic" disorder is present, however, fine-tuning of the normal-state Hamiltonian is nevertheless required for an odd-parity superconducting state to be perfectly immune to disorder, since in general the superconducting gap must be perfectly fit and the orbital-spin polarization of the Fermi surface must be vanishing.

An example of this fine-tuning has recently been provided in Ref. 25], which considers a purely Dirac system with $m=0, v_{z}=v$ and $\lambda=0$ in Eq. 34. By projecting the impurity potentials onto the band basis, it was found that odd-parity superconducting states may be completely robust against certain forms disorder. This result emerges straightforwardly within our framework, where the complete robustness is possible for the $A_{1 u}$ gap since it commutes with the remaining elements of the Hamiltonian (proportional to the $\gamma^{2}, \gamma^{3}$, and $\gamma^{4}$ matrices) and is therefore completely fit. For a general pairing potential, the effective scattering rate is

$$
\tilde{\tau}_{\nu}^{-1}=\sum_{\alpha} \pi n_{\mathrm{imp}, \alpha}\left|V_{\alpha}\right|^{2} \mathcal{N}\left[1-\lambda_{\alpha}\left(1-\tilde{F}_{C}\right)\right]
$$

where the superconducting fitness is easily evaluated since the three nonzero components of $\vec{\epsilon}_{\mathbf{k}}$ have equal magnitude on the (spherical) Fermi surface:

$$
\tilde{F}_{C}= \begin{cases}0 & A_{1 u} \\ 1 / 3 & \text { other odd parity }\end{cases}
$$

From this the magnitude of the effective scattering rates given in table III of Ref. 25] follows immediately.

\section{B. Even-parity states}

To conclude this section, we note that the analysis for the $s$-wave $A_{1 g}$ states is somewhat more complicated since the anomalous self-energy will generally always contain terms proportional to the conventional and unconventional pairing potentials. The general form for the $A_{1 g}$ gap is

$$
\Delta=\left[\Delta_{0}^{(0)} \gamma^{0}+\Delta_{0}^{(1)} \gamma^{1}\right] U_{T}
$$

and the corresponding lowest-order contribution to the anomalous self-energy is given by

$$
\Sigma_{2}^{(0)}=\sum_{\alpha} \pi n_{\mathrm{imp}, \alpha}\left|\tilde{V}_{\alpha}\right|^{2} \frac{\mathcal{N}}{2\left|\tilde{\omega}_{n}\right|}\left(\begin{array}{c}
\Delta_{0}^{(0)} \\
\Delta_{0}^{(1)}
\end{array}\right)^{\mathrm{T}}\left[\begin{array}{cc}
\lambda_{\alpha}^{(0)} & \lambda_{\alpha}^{(1)}\langle\hat{m}\rangle \\
\lambda_{\alpha}^{(0)}\langle\hat{m}\rangle & \lambda_{\alpha}^{(1)}\left\langle\hat{m}^{2}\right\rangle
\end{array}\right]\left(\begin{array}{c}
\gamma^{0} \\
\gamma^{1}
\end{array}\right) U_{T}
$$




\begin{tabular}{|c|c|c|c|c|c|c|c|c|c|c|c|c|c|c|c|c|c|}
\hline & & \multicolumn{16}{|c|}{$\tilde{V}_{\alpha}$} \\
\hline & & $\gamma^{0}$ & $\gamma^{1}$ & $\gamma^{2}$ & $\gamma^{3}$ & $\overline{\gamma^{4}}$ & $\gamma^{5}$ & $i \gamma^{1} \gamma^{2}$ & $i \gamma^{1} \gamma^{3}$ & $i \gamma^{1} \gamma^{4}$ & $i \gamma^{1} \gamma^{5}$ & $i \gamma^{2} \gamma^{3}$ & $i \gamma^{2} \gamma^{4}$ & $i \gamma^{2} \gamma^{5}$ & $i \gamma^{3} \gamma^{4}$ & $i \gamma^{3} \gamma^{5}$ & $i \gamma^{4} \gamma^{5}$ \\
\hline irrep & $\tilde{\Delta} U_{T}^{\dagger}$ & $\mathbb{q}_{4}$ & $\sigma_{0} \eta_{x}$ & $\sigma_{0} \eta_{y}$ & $\sigma_{x} \eta_{z}$ & $\sigma_{y} \eta_{z}$ & $\sigma_{z} \eta_{z}$ & $\sigma_{0} \eta_{z}$ & $\sigma_{x} \eta_{y}$ & $\sigma_{y} \eta_{y}$ & $\sigma_{z} \eta_{y}$ & $\sigma_{x} \eta_{x}$ & $\sigma_{y} \eta_{x}$ & $\sigma_{z} \eta_{x}$ & $\sigma_{z} \eta_{0}$ & $\sigma_{y} \eta_{0}$ & $\sigma_{x} \eta_{0}$ \\
\hline$A_{1 g}$ & $\gamma^{0}$ & +1 & +1 & -1 & -1 & -1 & -1 & +1 & +1 & +1 & +1 & -1 & -1 & -1 & -1 & -1 & -1 \\
\hline$A_{1 g}$ & $\gamma^{1}$ & +1 & +1 & +1 & +1 & +1 & +1 & -1 & -1 & -1 & -1 & -1 & -1 & -1 & -1 & -1 & -1 \\
\hline$A_{1 u}$ & $i \gamma^{1} \gamma^{5}$ & +1 & -1 & -1 & -1 & - & +1 & -1 & -1 & -1 & +1 & -1 & -1 & +1 & -1 & +1 & +1 \\
\hline$A_{2 u}$ & $i \gamma^{1} \gamma^{2}$ & +1 & -1 & +1 & -1 & -1 & -1 & +1 & -1 & -1 & -1 & +1 & +1 & +1 & -1 & -1 & -1 \\
\hline \multirow{2}{*}{$E_{u}$} & $i \gamma^{1} \gamma^{3}$ & +1 & -1 & -1 & +1 & -1 & -1 & -1 & +1 & -1 & -1 & +1 & -1 & -1 & +1 & +1 & -1 \\
\hline & $i \gamma^{1} \gamma^{4}$ & +1 & -1 & -1 & -1 & 于1 & -1 & -1 & 1 & +1 & -1 & -1 & +1 & -1 & +1 & -1 & +1 \\
\hline
\end{tabular}

Table I. The value of $\lambda_{\alpha}$ for the sixteen possible momentum-independent disorder potentials, for each of the six possible unconventional $s$-wave states, for $\mathrm{Cu}_{x} \mathrm{Bi}_{2} \mathrm{Se}_{3}$. This overall sign is calculated from $\tilde{V}_{\alpha} \tilde{\Delta} U_{T} \tilde{V}_{\alpha}^{\mathrm{T}}=\lambda_{\alpha} \tilde{\Delta} U_{T}$. Disorder potentials with $\lambda_{\alpha}=-1$ for the conventional $A_{1 g}$ gap $\left(\gamma^{0}\right)$ break time-reversal symmetry. The " $\otimes$ " in the orbital-spin form of the pairing and impurity potentials is omitted for clarity.

Because there is only a single band at the Fermi level, the effective scattering rate has the form

$$
\tilde{\tau}_{\nu}^{-1}=\tau^{-1}-\sum_{\alpha} \pi n_{\mathrm{imp}, \alpha}\left|V_{\alpha}\right|^{2} \mathcal{N}\left(\lambda_{\alpha}^{(0)}+\lambda_{\alpha}^{(1)}\langle\hat{m}\rangle^{2}\right)
$$

where $\lambda_{\alpha}^{(0)}$ and $\lambda_{\alpha}^{(1)}$ are the $\lambda$-factors for the conventional and unconventional states, respectively. We observe that $\phi_{\alpha}=\lambda_{\alpha}^{(0)} \lambda_{\alpha}^{(1)}$, from which it follows that the effective scattering rate is vanishing for $\lambda_{\alpha}^{(0)}=1$, i.e. the $s$-wave $A_{1 g}$ state is insensitive to disorder which preserves timereversal symmetry, as required by Anderson's theorem. The complete robustness of the unconventional $s$-wave state is due to the fact that the two gaps are indistinguishable on the single Fermi surface. As we will demonstrate in the following section, when both bands cross the Fermi energy the general gap becomes sensitive to time-reversal symmetry preserving disorder due to the unconventional component. This does not, however, violate Anderson's theorem as the unconventional component does not pair time-reversed partners.

\section{APPLICATION TO THE IRON PNICTIDES}

The framework we have presented can be applied in a straightforward way to systems with considerably more complicated Fermi surfaces than the single sheet Fermi surfaces of Dirac-like materials. To highlight this generality, we apply our method to a model for the iron pnictide superconductors for which two bands cross the Fermi level, each contributing two sheets to the Fermi surface.

We use a tight-binding model of the iron oxypnictides proposed by Raghu et al. 44, which includes only the contribution from the iron $d_{x z}$ and $d_{y z}$ orbitals. More sophisticated models, including up to five or more orbitals [48 50] better reproduce the electronic structure, but our focus here is in understanding the influence of the multiple Fermi surfaces, for which Raghu's model is sufficient.
The Hamiltonian for Raghu's model is written

$$
\begin{aligned}
\mathcal{H}_{\boldsymbol{k}}= & \varepsilon_{0}(\boldsymbol{k}) \sigma_{0} \otimes \tau_{0}+\varepsilon_{z}(\boldsymbol{k}) \sigma_{0} \otimes \tau_{z}+\varepsilon_{x}(\boldsymbol{k}) \sigma_{0} \otimes \tau_{x} \\
& +\lambda \sigma_{z} \otimes \tau_{y} \\
= & \varepsilon_{0}(\boldsymbol{k}) \gamma^{0}+\varepsilon_{z}(\boldsymbol{k}) \gamma^{1}+\varepsilon_{x}(\boldsymbol{k}) \gamma^{2}+\lambda \gamma^{5}
\end{aligned}
$$

with $\varepsilon_{0}=-\mu-\left(t_{1}+t_{2}\right)\left[\cos \left(k_{x}\right)+\cos \left(k_{y}\right)\right]-$ $4 t_{3} \cos \left(k_{x}\right) \cos \left(k_{y}\right), \varepsilon_{x}=-2 t_{4} \sin \left(k_{x}\right) \sin \left(k_{y}\right)$ and $\varepsilon_{z}=$ $-\left(t_{1}-t_{2}\right)\left[\cos \left(k_{x}\right)-\cos \left(k_{y}\right)\right]$. The Pauli matrices $\tau_{\nu}$ encode the iron $d_{x z}$ and $d_{y z}$ orbital degree of freedom, which transform trivially under inversion $(\mathcal{I}=\mathbb{1})$ and time-reversal. We extend the original model of Ref. 44 by including an additional spin-orbit coupling, in keeping with more general proposals [10, 31]. Throughout, we use the parameters $\left\{t_{1}, t_{2}, t_{3}, t_{4}, \mu\right\}=$ $\{-1,1.3,-0.85,-0.85,1.45\}\left|t_{1}\right|$ [44, and examine a variety of magnitudes of the spin-orbit coupling $\lambda$.

The normal-state scattering rates on the two bands are in general different and given by

$$
\begin{aligned}
\tau_{ \pm}^{-1}= & \sum_{\alpha} \frac{\pi n_{\mathrm{imp}, \alpha}\left|V_{\alpha}\right|^{2}}{2}\left[\mathcal{N}_{ \pm}\left(1+\phi_{\alpha}\langle\hat{\lambda}\rangle_{ \pm}^{2}\right)\right. \\
& \left.+\mathcal{N}_{\mp}\left(1-\phi_{\alpha}\langle\hat{\lambda}\rangle_{ \pm}\langle\hat{\lambda}\rangle_{\mp}\right)\right] .
\end{aligned}
$$

The second term in the brackets is the contribution from interband scattering. The non-zero Fermi surface average of the spin-orbit coupling gives a non-trivial dependence on the impurity potential. Specifically, for $\phi_{\alpha}=+1$, the spin-orbit coupling enhances intraband scattering and suppresses interband scattering, whereas $\phi_{\alpha}=-1$ gives the opposite effect. This result can be easily understood in the extreme limit $\hat{\lambda} \rightarrow 1$ where the two bands become eigenstates of the spin-orbit coupling operator $\gamma^{5}$. Scattering off a disorder potential which commutes with $\gamma^{5}$ (and therefore has $\phi_{\alpha}=1$ ) does not change the $\gamma^{5}$-eigenstate of the electron, and hence cannot scatter between the two bands. Similarly, disorder that anti-commutes with the spin-orbit coupling operator is incapable of intraband scattering in this extreme 
limit. On the other hand, when $\lambda=0$ there is no net spin-orbital polarization of either band, and the scattering rates in the two bands become indistinguishable.

Due to the trivial inversion symmetry, the unconventional $s$-wave states have even parity. As tabulated in Tab. II. the unconventional $s$-wave states belong to the $A_{1 g}, B_{1 g}, B_{2 g}$ and $E_{g}$ irreps of the $D_{4 h}$ point group. From Eq. 14 we observe that the $E_{g}$ gaps are completely unfit since the two-dimensional Hamiltonian does not contain any terms proportional to $\gamma^{3}$ and $\gamma^{4}$, and so we will not consider the $E_{g}$ states in the following. Since the $s$-wave pairing states are all even-parity, their projections into a pseudospin basis is explicitly given by

$$
\begin{aligned}
\Delta_{0} \gamma^{0} U_{T} \rightarrow \Delta_{ \pm} & =\Delta_{0} i s_{y} \\
\Delta_{0} \gamma^{1} U_{T} \rightarrow \Delta_{ \pm} & = \pm \hat{\epsilon}_{z} \Delta_{0} i s_{y} \\
\Delta_{0} \gamma^{2} U_{T} \rightarrow \Delta_{ \pm} & = \pm \hat{\epsilon}_{x} \Delta_{0} i s_{y} \\
\Delta_{0} \gamma^{5} U_{T} \rightarrow \Delta_{ \pm} & = \pm \hat{\lambda} \Delta_{0} i s_{y}
\end{aligned}
$$

Note that the unconventional $A_{1 g}$ state has an $s^{ \pm}$form, with a full gap with opposite signs on the electron- and hole-like Fermi surfaces. This is widely accepted as the sign structure of the pairing state in the iron pnictides, although it is important to emphasize that this can be achieved with an orbitally-trivial pairing potential, as we discuss below.

\section{A. The $B_{1 g}$ and $B_{2 g}$ irreps}

We begin by considering the $B_{1 g}$ and $B_{2 g}$ irreps, which both have a single unconventional $s$-wave pairing potential. We first examine the robustness of the unconventional $s$-wave state, and then compare this against orbitally-trivial $d$-wave spin-singlet pairing states in the same irrep.

\section{1. s-wave gaps}

The usual Abrikosov-Gor'kov result for the suppression of an unconventional pairing state in a two-band system is

$$
\log \left(\frac{T_{c}}{T_{c 0}}\right)=\sum_{j= \pm} R_{j}\left[\psi\left(\frac{1}{2}\right)-\psi\left(\frac{1}{2}+\frac{1}{4 \pi k_{B} T_{c} \tau_{j}}\right)\right],
$$

where the contribution of the $j$ th band is weighted according to its contribution to the condensation energy

$$
R_{j}=\frac{\mathcal{N}_{j}\left\langle\left|\tilde{\Delta}_{\boldsymbol{k}}\right|^{2}\right\rangle_{j}}{\mathcal{N}_{+}\left\langle\left|\tilde{\Delta}_{\boldsymbol{k}}\right|^{2}\right\rangle_{+}+\mathcal{N}_{-}\left\langle\left|\tilde{\Delta}_{k}\right|^{2}\right\rangle_{-}} .
$$

This result naturally reduces to the single-band case in the limit where one of the densities of states vanishes. Moreover, in the absence of the spin-orbit coupling the normal-state scattering rates are independent of the band index, i.e. $\tau_{ \pm}^{-1}=\tau^{-1}$, and we recover the usual universal result.

Accounting for the nontrivial orbital-spin structure of the unconventional $s$-wave pairing state in channel $\nu$, the expression Eq. 49 is modified as $\tau_{j}^{-1} \rightarrow \bar{\tau}_{\nu, j}^{-1}$ and $R_{j} \rightarrow$ $\bar{R}_{j}$. The effective scattering rate $\bar{\tau}_{\nu, j}^{-1}$ is given by

$$
\bar{\tau}_{\nu, \pm}^{-1}=\frac{\tilde{\tau}_{\nu,+}^{-1}+\tilde{\tau}_{\nu,-}^{-1}}{2} \pm \sqrt{\left[\frac{\tilde{\tau}_{\nu,+}^{-1}-\tilde{\tau}_{\nu,-}^{-1}}{2}\right]^{2}+\left(\tilde{\tau}_{\nu,+}^{-1}-\tau_{+}^{-1}\right)\left(\tilde{\tau}_{\nu,-}^{-1}-\tau_{-}^{-1}\right)}
$$

with

$$
\tilde{\tau}_{\nu, j}^{-1}=\tau_{j}^{-1}-\sum_{\alpha} \pi \lambda_{\alpha} n_{\mathrm{imp}, \alpha}\left|V_{\alpha}\right|^{2} \mathcal{N}_{j}\left(1-\tilde{F}_{C}^{(j)}\right) .
$$

We recognize the $\tilde{\tau}_{\nu, j}^{-1}$ as the generalization of Eq. 36 to the multiband case, assuming that we can treat each band independently. However, the unconventional $s$ wave pairing potentials couple the two bands, and we therefore cannot readily associate the scattering rates in Eq. 51 with one band or the other. The weighting of the contribution from the two effective scattering rates also deviates from the expected form Eq. 50, and depends upon the normal-state and effective scattering rates

$$
\bar{R}_{ \pm}=R_{ \pm} \pm \frac{\mathcal{N}_{+}\left\langle\left|\tilde{\Delta}_{k}\right|^{2}\right\rangle_{+}\left(\bar{\tau}_{\nu,-}^{-1}-\tau_{-}^{-1}\right)+\mathcal{N}_{-}\left\langle\left|\tilde{\Delta}_{k}\right|^{2}\right\rangle_{-}\left(\bar{\tau}_{\nu,+}^{-1}-\tau_{+}^{-1}\right)}{\left[\mathcal{N}_{+}\left\langle\left|\tilde{\Delta}_{k}\right|^{2}\right\rangle_{+}+\mathcal{N}_{-}\left\langle\left|\tilde{\Delta}_{\boldsymbol{k}}\right|^{2}\right\rangle_{-}\right]\left(\bar{\tau}_{\nu,+}^{-1}-\bar{\tau}_{\nu,-}^{-1}\right)}
$$




\begin{tabular}{|c|c|c|c|c|c|c|c|c|c|c|c|c|c|c|c|c|c|}
\hline & & \\
\hline & & $\gamma^{0}$ & $\gamma^{1}$ & $\gamma^{2}$ & $\gamma^{3}$ & $\gamma^{4}$ & $\gamma^{5}$ & $i \gamma^{1} \gamma^{2}$ & $i \gamma^{1} \gamma^{3}$ & $i \gamma^{1} \gamma^{4}$ & $i \gamma^{1} \gamma^{5}$ & $i \gamma^{2} \gamma^{3}$ & $i \gamma^{2} \gamma^{4}$ & $i \gamma^{2} \gamma^{5}$ & $i \gamma^{3} \gamma^{4}$ & $i \gamma^{3} \gamma^{5}$ & $i \gamma^{4} \gamma^{5}$ \\
\hline rrep & $\tilde{\Delta} U_{T}^{\dagger}$ & $\mathbb{0}_{4}$ & $\sigma_{0} \eta_{z}$ & $\sigma_{0} \eta_{x}$ & $\sigma_{x} \eta_{y}$ & $\sigma_{y} \eta_{y}$ & $\sigma_{z} \eta_{y}$ & $\sigma_{0} \eta_{y}$ & $\sigma_{x} \eta_{x}$ & $\sigma_{y} \eta_{x}$ & $\sigma_{z} \eta_{x}$ & $\sigma_{x} \eta_{z}$ & $\sigma_{y} \eta_{z}$ & $\sigma_{z} \eta_{z}$ & $\sigma_{z} \eta_{0}$ & $\sigma_{y} \eta_{0}$ & $\sigma_{x} \eta_{0}$ \\
\hline$A_{1 g}$ & $\gamma^{0}$ & +1 & +1 & +1 & +1 & +1 & +1 & -1 & -1 & -1 & -1 & -1 & -1 & -1 & -1 & -1 & -1 \\
\hline$B_{1 g}$ & $\gamma^{1}$ & +1 & +1 & -1 & -1 & -1 & -1 & +1 & +1 & +1 & +1 & -1 & -1 & -1 & -1 & -1 & -1 \\
\hline$B_{2 g}$ & $\gamma^{2}$ & +1 & -1 & +1 & -1 & -1 & -1 & +1 & -1 & -1 & -1 & +1 & +1 & +1 & -1 & -1 & -1 \\
\hline \multirow{2}{*}{$E_{g}$} & $\gamma^{3}$ & +1 & -1 & -1 & +1 & -1 & -1 & - & + & -1 & - & +1 & -1 & -1 & +1 & +1 & -1 \\
\hline & $\gamma^{4}$ & +1 & -1 & -1 & -1 & +1 & -1 & - & - & +1 & - & - & +1 & -1 & +1 & -1 & +1 \\
\hline $4_{1 g}$ & $\gamma^{5}$ & +1 & -1 & -1 & -1 & -1 & +1 & -1 & -1 & -1 & +1 & -1 & -1 & +1 & -1 & +1 & +1 \\
\hline
\end{tabular}

Table II. The value of $\lambda_{\alpha}$ for the sixteen possible momentum-independent disorder potentials, for each of the six possible unconventional $s$-wave states for the model of iron pnictide superconductors proposed by Raghu et al. 44]. Disorder potentials with $\lambda_{\alpha}=-1$ for the first $A_{1 g}$ gap, proportional to the identity matrix, break time-reversal symmetry.

Using these expressions, we plot the critical temperature of the $B_{1 g}$ and $B_{2 g} s$-wave gaps as a function of the strength of "nonmagnetic" and "magnetic" disorder in Figs. 2 and 3 , respectively. Notably, the $B_{1 g}$ state is extremely robust against "nonmagnetic" disorder, with superconductivity persisting to a disorder strength more than ten times that expected from Eq. 49 in contrast, the $B_{2 g}$ state closely follows the predictions of the AbrikosovGor'kov theory.

The pronounced robustness of the $B_{1 g}$ state is due to the almost-perfect fitness on the Fermi surface of the + band. For realistic values of the spin-orbit coupling, on this Fermi surface we have $\varepsilon_{z} \gg \varepsilon_{x}, \lambda$, and so $F_{C}^{(+)}=$ $1-\left\langle\hat{\varepsilon}_{z}^{2}\right\rangle_{+} \approx 0$; on the other hand, the average fitness on the Fermi surfaces of the - band is much smaller, with the gap displaying nodes along the Brillouin zone diagonals where the potential is completely unfit. In the limit where the spin-orbit coupling is vanishing, we find that the effective scattering rates are

$$
\bar{\tau}_{\nu, \pm}^{-1}=\frac{\pi}{4} \sum_{j} \sum_{\alpha} n_{\mathrm{imp}, \alpha}\left|V_{\alpha}\right|^{2} \mathcal{N}_{j}\left(2 \pm\left[1 \mp \lambda_{\alpha}\right]\left[1-F_{C}^{(j)}\right]\right) .
$$

Taking $F_{C}^{(+)} \approx 0$, and restricting to "nonmagnetic" disorder $\left(\lambda_{\alpha}=+1\right)$ we find that $\bar{\tau}_{\nu,+}^{-1} \approx \tau^{-1}$ and $\bar{\tau}_{\nu,-}^{-1} \approx$ $\pi \sum_{\alpha} n_{\text {imp }, \alpha}\left|V_{\alpha}\right|^{2} \mathcal{N}_{-} F_{C}^{(-)}$. Since $\mathcal{N}_{+} \gg \mathcal{N}_{-}$in Raghu's model, we expect that $\bar{\tau}_{\nu,-}^{-1} \ll \tau^{-1}$. Inserting these expressions into Eq. 53 we find that $\bar{R}_{+} \approx 0$ and $\bar{R}_{-} \approx 1$. We thus see that critical temperature is controlled by one effective SR $\bar{\tau}_{\nu,-}^{-1}$, which can be much smaller than the normal-state SR. Note that due to the almost-perfect fitness of the superconductivity on the Fermi surfaces of the + band, the disorder-response is completely controlled by the fitness on the Fermi surfaces of the - band.

The comparable fragility of the $B_{2 g}$ gap can also be understood using these arguments. The $B_{2 g}$ gap is much less fit than the $B_{1 g}$ gap on the Fermi surface of the + band, but has comparable fitness on the Fermi surfaces of the - band. Repeating the analysis above, but now taking $\tilde{F}_{C}^{(+)} \approx 1$, we find that $\bar{\tau}_{\nu,+}^{-1} \approx \tau^{-1}$ and $\bar{\tau}_{\nu,-}^{-1} \approx$

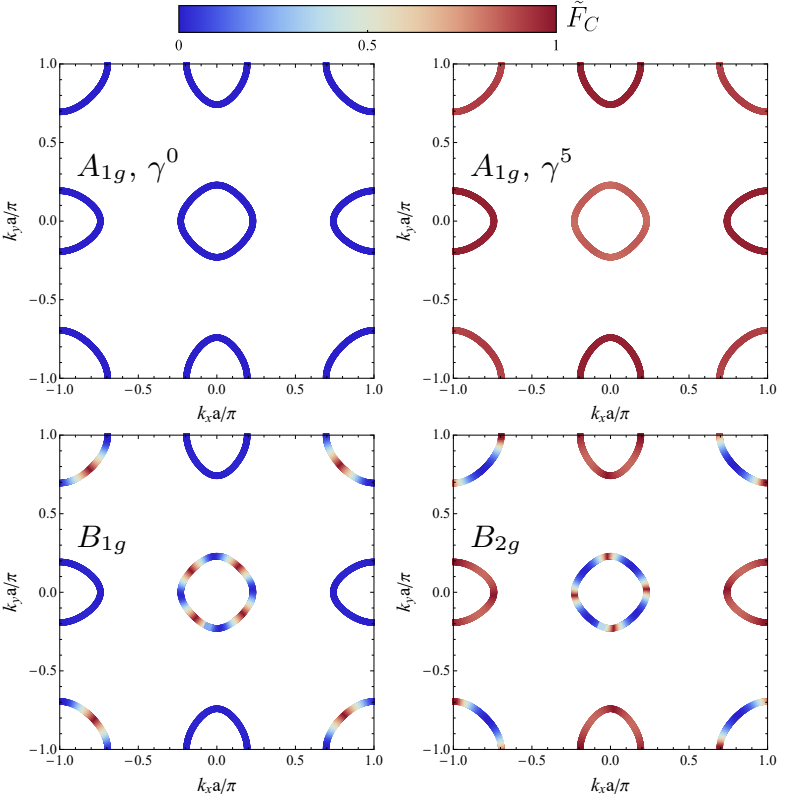

Figure 1. Fermi surface for the two-orbital model for iron pnictides, with $\lambda=0.1\left|t_{1}\right|$, and superconducting fitness for the four $s$-wave states considered. The coloring of the contours denotes the magnitude of the superconducting fitness. For the conventional $A_{1 g}$ state $\left(\gamma^{0}\right)$, the fitness is zero everywhere, while for the unconventional state $\left(\gamma^{5}\right)$ it varies between the bands. The $B_{1 g}$ state also has vanishing averaged fitness on the + band Fermi surface, while the $B_{2 g}$ fitness is finite everywhere on the Fermi surface.

$\tau^{-1}-\pi \sum_{\alpha} n_{\text {imp }, \alpha}\left|V_{\alpha}\right|^{2} \mathcal{N}_{-}\left[1-F_{C}^{(-)}\right]$. For the parameters of Raghu's model, the two effective scattering rates are similar, and the suppression of the pairing by disorder is therefore well approximated by the Abrikosov-Gor'kov result. 

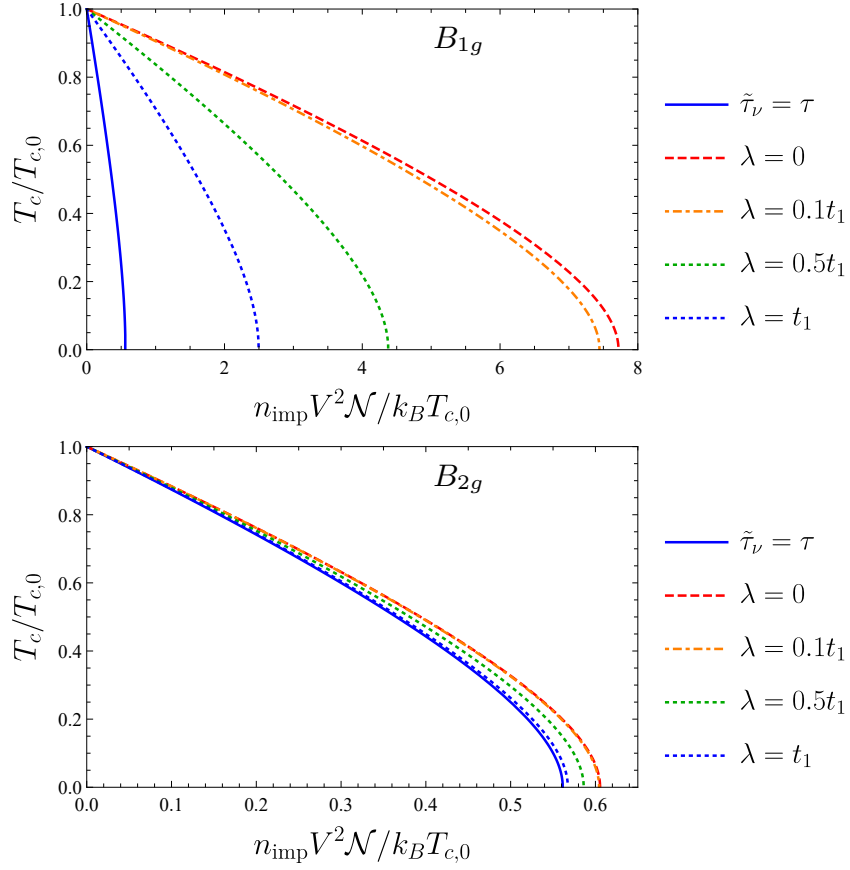

Figure 2. Robustness against "nonmagnetic" $\left(\lambda_{\alpha}=+1\right)$ disorder for the $B_{1 g}$ (top) and $B_{2 g}$ (bottom) $s$-wave gaps in the two-orbital model of the iron pnictides, for various values of the spin-orbit coupling $\lambda$.

\section{2. d-wave gaps}

The $B_{1 g}$ and $B_{2 g} s$-wave states have $d$-wave formfactors when projected onto the states at the Fermi surface. We have previously demonstrated [19] that momentum-dependent pairing states that belong to an irrep with an unconventional $s$-wave state will generally inherit some of the robustness of that $s$-wave state again disorder, due to overlap of the two gaps at the Fermi surface. Solving the linearized gap equation for a multi-component gap, the generalization of Eq. 49 for momentum-dependent gaps is

$$
\begin{aligned}
\log \left(\frac{T_{c}}{T_{c 0}}\right)= & \sum_{j= \pm} \bar{R}_{j}\left[\psi\left(\frac{1}{2}\right)-\psi\left(\frac{1}{2}+\frac{1}{4 \pi k_{B} T_{c} \bar{\tau}_{\nu, j}}\right)\right] \\
& +\sum_{j= \pm} R_{j}\left[\psi\left(\frac{1}{2}\right)-\psi\left(\frac{1}{2}+\frac{1}{4 \pi k_{B} T_{c} \tau_{j}}\right)\right],
\end{aligned}
$$

where the first line accounts for the overlap with the unconventional $s$-wave states. The parameters $\bar{R}_{j}$ in this expression are reduced compared to those of the purely $s$-wave case, as they satisfy $\sum_{j= \pm} R_{j}+\bar{R}_{j}=1$.

Equation 55 is distinguished by the presence of more than one effective scattering rate, which can significantly alter the shape of the disorder curve. This can be understood more concretely by considering the limiting behavior of Eq. 55 at both strong and weak disorder, where the curve is characterized by a single effective scattering rate. A Taylor expansion in the weak disorder limit for Eq. 55 gives $T_{c} \approx T_{c, 0}-\pi / 8 k_{B} \tau_{\mathrm{WD}}$, dependent on a single average effective scattering rate

$$
\tau_{\mathrm{WD}}^{-1}=\sum_{i= \pm} \bar{R}_{i} \bar{\tau}_{i}^{-1}+R_{i} \tau_{i}^{-1}
$$

while for strong disorder the critical temperature can be shown to vanish at a disorder strength given by the usual expression [1, 51] $\log \left(2 \pi k_{B} T_{c, 0} \tau_{\mathrm{SD}}\right)=-\psi(1 / 2)$ with an effective scattering rate

$$
\tau_{\mathrm{SD}}^{-1}=\prod_{i= \pm}\left(\bar{\tau}_{i}^{-1}\right)^{\bar{R}_{i}}\left(\tau_{i}^{-1}\right)^{R_{i}} .
$$

In general for a multi-band system $\tau_{\mathrm{WD}} \neq \tau_{\mathrm{SD}}$, except when only a single scattering rate is present, such as for an unconventional $s$-wave state with a single band at the Fermi level.

To illustrate, we consider an orbitally-trivial $d$-wave $B_{1 g}$ state in the two-band iron pnictide model with $\Delta=\Delta_{0}\left(\cos k_{x}-\cos k_{y}\right) \gamma^{0} U_{T} / 2$. The lowest-order contribution to the anomalous self-energy for this gap is proportional to the corresponding unconventional $s$-wave potential

$$
\begin{aligned}
\Sigma_{2}^{(0)}= & \Delta_{0} \sum_{\alpha} \pi n_{\mathrm{imp}, \alpha}\left|\tilde{V}_{\alpha}\right|^{2} \lambda_{\alpha}^{(1)} \\
& \times \sum_{j= \pm} \frac{j \mathcal{N}_{j}}{4\left|\tilde{\omega}_{n, j}\right|}\left\langle\left(\cos k_{x}-\cos k_{y}\right) \varepsilon_{z}\right\rangle_{j} \gamma^{1} U_{T} .
\end{aligned}
$$

Since $\varepsilon_{z}$ also belongs to $B_{1 g}$, the Fermi surface average in this expression - the overlap between the $d$-wave and $s$-wave states - is generally nonzero. We plot the critical temperature against the "nonmagnetic" (with respect to the $s$-wave $B_{1 g}$ state) disorder strength for this state in Fig. 4. The $d$-wave state is considerably less robust against "nonmagnetic" disorder than the $s$-wave state, but still much more robust than predicted by the Abrikosov-Gor'kov result (solid line). The shape of the curve also differs noticeably from the typical AbrikosovGor'kov curve, in particular for weak spin-orbit coupling: while the critical temperature is initially suppressed linearly with disorder, the gradient decreases with increasing disorder, and clearly $\tau_{\mathrm{WD}}^{-1}>\tau_{\mathrm{SD}}^{-1}$. The robustness of the $d$-wave state against "magnetic" disorder, included in Fig. 3 is again influenced by the overlap with the unconventional $s$-wave state, which in this case reduces the robustness relative to a single-band $d$-wave gap.

\section{B. The $A_{1 g}$ irrep}

As was the case for the Dirac system considered above, the behavior of the two-channel $A_{1 g}$ state is more complicated than that of the single channel superconducting states. The general pairing potential in this state is given by

$$
\Delta=\left[\Delta_{0}^{(0)} \gamma^{0}+\Delta_{0}^{(5)} \gamma^{5}\right] U_{T}
$$




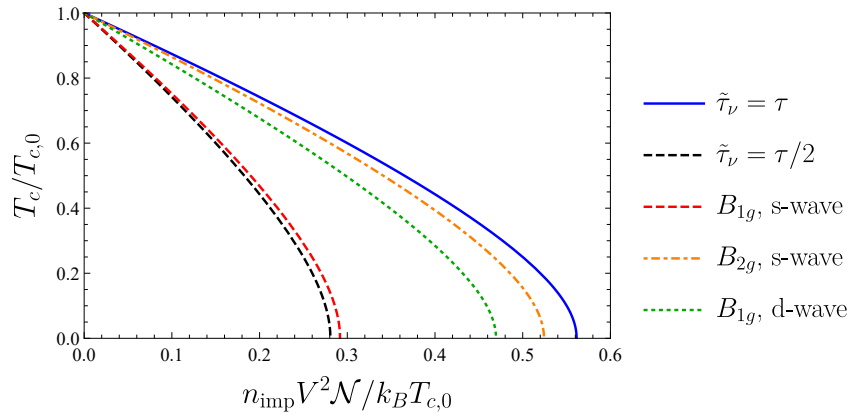

Figure 3. Robustness against "magnetic" $\left(\lambda_{\alpha}=-1\right)$ disorder for the $s$-wave $B_{1 g}$ and $B_{2 g}$ gaps, as well as the $d$-wave $B_{1 g}$ gap, in the two-orbital model of the iron pnictides, with spinorbit coupling $\lambda=0.1\left|t_{1}\right|$.

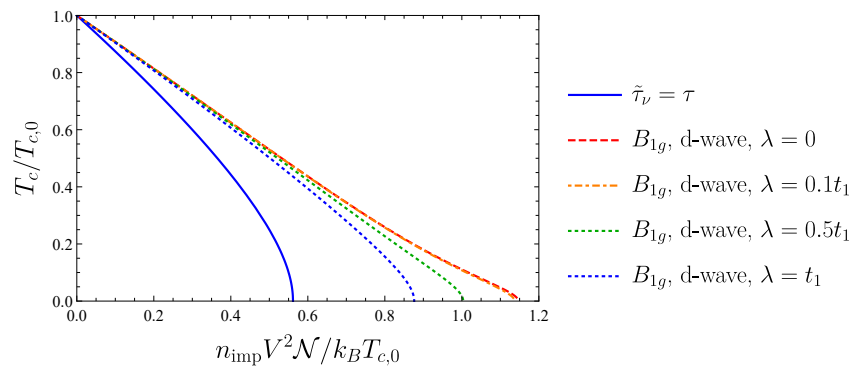

Figure 4. Robustness against "nonmagnetic" $\left(\lambda_{\alpha}=+1\right)$ disorder for $B_{1 g} d$-wave gaps in the two-orbital model of the iron pnictides, for various values of the spin-orbit coupling $\lambda$. where any combination of the two $s$-wave channels is allowed. Projected into the band-pseudospin basis, we have

$$
\Delta \rightarrow \Delta_{ \pm}=\left(\Delta_{0}^{(0)} \pm \hat{\lambda} \Delta_{0}^{(5)}\right) i s_{y}
$$

This state is typically fully-gapped, and in the presence of spin-orbit coupling it will change sign between the + and - bands when the ratio $\Delta_{0}^{(0)} / \Delta_{0}^{(5)}$ is sufficiently small.

In general we can have attractive interactions in both the conventional and unconventional channels, which we label $g_{0}$ and $g_{5}$, respectively. The critical temperature $T_{c}$ of the mixed pairing state has a complicated form complicated expression, but for realistic parameters we find that the actual $T_{c}$ is very close to the greater of $T_{c, 0}^{(0)}$ and $T_{c, 0}^{(5)}$, which are the critical temperatures of each channel in the absence of the other. These have the explicit form

$$
\begin{aligned}
& T_{c, 0}^{(0)}=\frac{2 e^{\gamma}}{\pi} \Lambda \exp \left(-\frac{1}{g_{0}\left[\mathcal{N}_{+}+\mathcal{N}_{-}\right]}\right) \\
& T_{c, 0}^{(5)}=\frac{2 e^{\gamma}}{\pi} \Lambda \exp \left(-\frac{1}{g_{5}\left[\left\langle\hat{\lambda}^{2}\right\rangle_{+} \mathcal{N}_{+}+\left\langle\hat{\lambda}^{2}\right\rangle_{-} \mathcal{N}_{-}\right]}\right)
\end{aligned}
$$

where $\Lambda$ is a cut-off and $\gamma$ is Euler's constant.

The lowest-order contribution to the anomalous selfenergy is

$$
\Sigma_{2}^{(0)}=\sum_{\alpha} \pi n_{\mathrm{imp}, \alpha}\left|\tilde{V}_{\alpha}\right|^{2} \sum_{j= \pm} \frac{\mathcal{N}_{j}}{2\left|\tilde{\omega}_{n, j}\right|}\left(\begin{array}{c}
\Delta_{0}^{(0)} \\
\Delta_{0}^{(5)}
\end{array}\right)^{\mathrm{T}}\left[\begin{array}{cc}
\lambda_{\alpha}^{(0)} & \lambda_{\alpha}^{(5)} j\langle\hat{\lambda}\rangle_{j} \\
\lambda_{\alpha}^{(0)} j\langle\hat{\lambda}\rangle_{j} & \lambda_{\alpha}^{(5)}\left\langle\hat{\lambda}^{2}\right\rangle_{j}
\end{array}\right]\left(\begin{array}{c}
\gamma^{0} \\
\gamma^{5}
\end{array}\right) U_{T}
$$

where we see that the disorder only couples the two channels if the spin-orbit coupling is nonzero. This expression is similar to the self-energy for the $A_{1 g}$ states in $\mathrm{Cu}_{x} \mathrm{Bi}_{2} \mathrm{Se}_{3} \mathrm{Eq}$. 41, where the conventional and unconventional $s$-wave $A_{1 g}$ states are only coupled when the mass $m$ is nonzero. In contrast with the case of $\mathrm{Cu}_{x} \mathrm{Bi}_{2} \mathrm{Se}_{3}$, however, the general $A_{1 g}$ state is not completely robust against "nonmagnetic" disorder. This is a direct consequence of the presence of both bands at the Fermi energy and the changing sign of the unconventional gap component between the two bands. The general expression for the critical temperature is lengthy and will not be presented here; numerical solutions of the linearized gap equation are plotted in Figs. 5 and 6 .

\section{Purely unconventional pairing}

In the limit that the pairing potential vanishes in the conventional $s$-wave channel, $g_{0}=0$, the superconducting gap is purely unconventional, with equal magnitude and opposite sign on the two Fermi surfaces. The critical temperature for the purely unconventional state is given by Eq. 49, with one of the two effective scattering rates vanishing in the presence of TRS disorder, $\bar{\tau}_{-}^{-1}=0$,

$$
\log \left(\frac{T_{c}}{T_{c 0}}\right)=\bar{R}_{+}\left[\psi\left(\frac{1}{2}\right)-\psi\left(\frac{1}{2}+\frac{1}{4 \pi k_{B} T_{c} \bar{\tau}_{+}}\right)\right] .
$$

Significantly, despite the absence of a contribution due to $\bar{\tau}_{-}$, the parameter $\bar{R}_{-}$, which characterizes the overlap of the unconventional $s$-wave state with the conventional, is non-vanishing, and as such $\bar{R}_{+}<1$. Specifically, for TRS 
$\operatorname{disorder}\left(\lambda_{\alpha}^{(0)}=+1\right)$

$\begin{aligned} \bar{R}_{+} & =\frac{\mathcal{N}_{+} \mathcal{N}_{-}\left(\langle\lambda\rangle_{+}+\langle\lambda\rangle_{-}\right)^{2}}{\left(\mathcal{N}_{+}+\mathcal{N}_{-}\right)\left(\mathcal{N}_{+}\left\langle\lambda^{2}\right\rangle_{+}+\mathcal{N}_{-}\left\langle\lambda^{2}\right\rangle_{-}\right)} \\ \bar{\tau}_{+}^{-1} & =\frac{\pi \sum_{\alpha} n_{\text {imp }, \alpha}\left|V_{\alpha}\right|^{2}}{2}\left(\mathcal{N}_{+}+\mathcal{N}_{-}\right)\left(1-\lambda_{\alpha}^{(5)}\langle\lambda\rangle_{+}\langle\lambda\rangle_{-}\right),\end{aligned}$

and the effective scattering rate is simply the total interband scattering rate.

For weak disorder, the suppression of $T_{c}$ is linear in $\bar{\tau}_{+}^{-1}$, but in the strong disorder limit, we find

$$
T_{c} \propto \tau_{0}^{\left(\frac{1-\bar{R}_{-}}{R_{-}}\right)} \sim|V|^{2\left(\frac{\bar{R}_{-}-1}{R_{-}}\right)}
$$

and $T_{c}$ is exponentially suppressed for $0<\bar{R}_{-}<1$. Interestingly, we find that even in the complete absence of conventional pairing the superconductivity retains some residual robustness against TRS disorder, due to the overlap with the conventional state. This effect is evident in the small exponential tail in the strong disorder limit for the purely unconventional curve in Fig. 5 close to $T_{c}=0$. For the particular model parameters adopted here we find that $\tilde{R}_{-} \ll 1$, and so for strong disorder $T_{c}$ is nearly indistinguishable from zero for the purely unconventional pairing state.

In contrast, for TRSB disorder, $\bar{\tau}_{ \pm}^{-1} \neq 0$, and the superconductivity is completely suppressed for strong disorder. In fact, the influence of the conventional $s$-wave state is slightly detrimental to the purely unconventional $s$-wave state (see Fig. 5), due to the greater sensitivity of the conventional gap to TRSB disorder.

\section{Coexisting conventional and unconventional pairing}

In the presence of time-reversal symmetry preserving disorder (with $\lambda_{\alpha}^{(0)}=+1$ for the conventional gap component), any general mixture of the two channels with $T_{c, 0}^{(5)}<T_{c, 0}^{(0)}$ is completely robust, whereas a state with $T_{c, 0}^{(5)}>T_{c, 0}^{(0)}$ is sensitive to disorder. In the latter case, the critical temperature follows closely the curve of the purely unconventional state at weak disorder, but saturates at the critical temperature $T_{c, 0}^{(0)}$ of the purely conventional state in the strong-disorder limit. As seen in Fig. 6. this crossover occurs when the critical temperature for the purely-unconventional state falls below that of the purely-conventional state, with the critical temperature of the mixed state closely tracking the higher of the two. This can hence be interpreted as a disorder-induced crossover from a state where the unconventional pairing dominates to a state where the conventional pairing is dominant. This crossover between $s^{ \pm}$-wave and conventional $s$-wave states has been extensively studied in models where the orbital degree of freedom is not explicitly included [37, 38, 41].
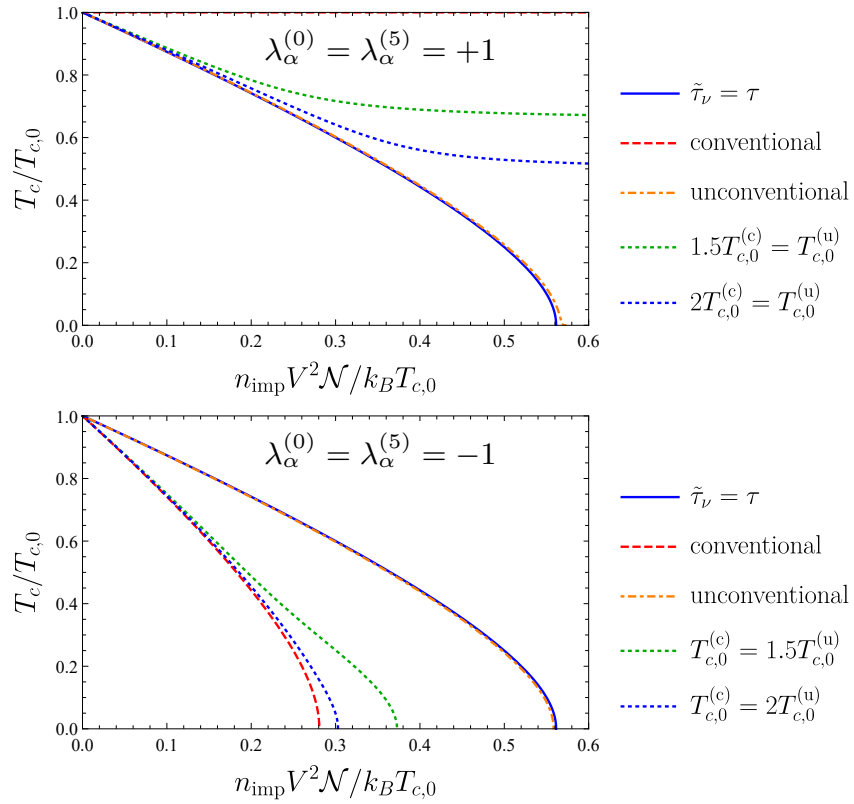

Figure 5. Robustness of the multi-channel $s$-wave $A_{1 g}$ gap against against TRS (top) and TRSB (bottom) disorder, in the two-orbital model for iron oxypnictides with weak spinorbit coupling $\lambda=0.1\left|t_{1}\right| . \lambda_{\alpha}^{(0)}$ is defined for the conventional $s$-wave state, and we find that the influence of $\lambda_{\alpha}^{(5)}$ for the unconventional gap is minimal. The states with pairing in both channels are labeled by the relative critical temperatures in the clean limit for the conventional $\left(T_{c, 0}^{(\mathrm{c})}\right)$ and unconventional $\left(T_{c, 0}^{(\mathrm{u})}\right)$ channels.

For time-reversal symmetry breaking disorder potentials $\left(\lambda_{\alpha}^{(0)}=-1\right)$ there may alternatively exist a crossover in the intermediate disorder strength regime when $T_{c, 0}^{(5)}<$ $T_{c, 0}^{(0)}$. As shown in Fig. 6 this crossover is less general than that for TRS disorder, since it requires that the unconventional state is sufficiently competitive with the conventional and has sufficiently small effective scattering rate, so that the termination point of the purelyunconventional state is at stronger disorder strength. If the unconventional state dominates in the clean limit (i.e. $\left.T_{c, 0}^{(5)}>T_{c, 0}^{(0)}\right)$, the critical temperature closely tracks the curve for purely-unconventional pairing, and no crossover is observed. These contrasting crossover effects could be used to evidence the dominant component in the clean limit of a mixed pairing state.

\section{The momentum-dependent $s_{ \pm}$-wave $A_{1 g}$ gap}

Finally, we turn our attention to a momentumdependent $s_{ \pm}$-wave singlet state,

$$
\Delta=\Delta_{0} \cos \left(k_{x}\right) \cos \left(k_{y}\right) U_{T} .
$$

The form factor ensures that the gap on the + and Fermi surfaces has opposite sign, similar to the uncon- 


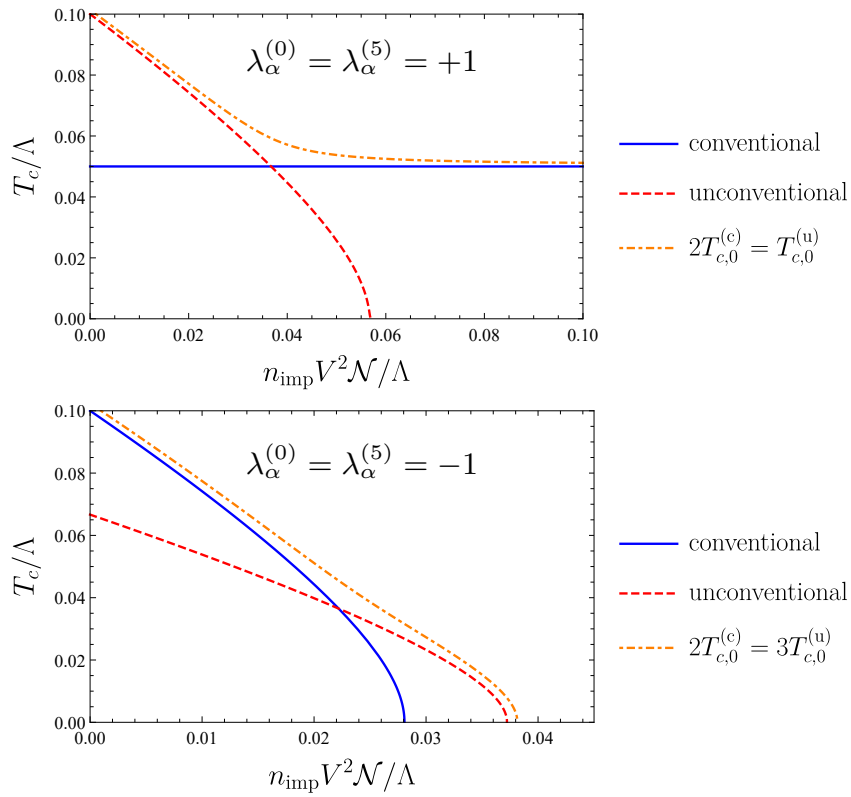

Figure 6. Robustness of the multi-channel $s$-wave $A_{1 g}$ gap against against 'nonmagnetic' $\left(\lambda_{\alpha}^{(0)}=\lambda_{\alpha}^{(5)}=+1\right.$, top) and 'magnetic' $\left(\lambda_{\alpha}^{(0)}=\lambda_{\alpha}^{(5)}=-1\right.$, bottom $)$ disorder, in the twoorbital model for iron oxypnictides with weak spin-orbit coupling $\lambda=0.1\left|t_{1}\right|$. Unlike Fig. 5 both critical temperature and disorder strength are scaled by $\Lambda$, rather than $T_{c, 0}$ (with $T_{c, 0}^{(\mathrm{c})}=0.1 \Lambda$ and $\left.T_{c, 0}^{(\mathrm{u})}=0.05 \Lambda\right)$. ventional $s$-wave state. In this case, the robustness depends on the overlap of the momentum-dependent state, with $f_{\boldsymbol{k}}=\cos \left(k_{x}\right) \cos \left(k_{y}\right)$, and both $A_{1 g} s$-wave states, as well as the robustness of those states and the effect of the normal state scattering rate. The lowest-order contribution to the anomalous self-energy is defined by the overlap of the $s_{ \pm}$-wave state with the conventional and unconventional $s$-wave states,

$$
\Sigma_{2}^{(0)}=\pi \sum_{\alpha} \frac{n_{\mathrm{imp}, \alpha}\left|\tilde{V}_{\alpha}\right|^{2}}{2} \sum_{j= \pm}\left[\lambda_{\alpha}^{(0)} \frac{\mathcal{N}_{j}\left\langle f_{\boldsymbol{k}}\right\rangle_{j}}{\left|\tilde{\omega}_{n, j}\right|} \gamma^{0}+j \lambda_{\alpha}^{(5)} \frac{\mathcal{N}_{j}\left\langle f_{\boldsymbol{k}} \hat{\lambda}\right\rangle_{j}}{\left|\tilde{\omega}_{n, j}\right|} \gamma^{5}\right] U_{T}
$$

and the overlap with the unconventional $s$-wave state depends on the spin-orbit coupling magnitude $\lambda$. Ultimately, the critical temperature is given by an expression of the form Eq. 55, with one effective scattering rate vanishing $\bar{\tau}_{-}^{-1}=0$ for TRS disorder.

As in the case of the purely unconventional $s$-wave state, $T_{c}$ is exponentially suppressed, as described by Eq. 66, for strong TRS disorder, but the much greater overlap of the $s_{ \pm}$-wave gap with the conventional $s$-wave enhances this effect.

Spin-orbital effects play a significant role in determining the robustness of the $s_{ \pm}$-wave state. Increasing the spin-orbit coupling $\lambda$ increases both the interband scattering and, more significantly, the overlap with the unconventional $s$-wave state. Unlike the momentumdependent $B_{1 g} d$-wave state, the existence of the unconventional $s$-wave state is detrimental to the robustness of the momentum dependent $A_{1 g} s_{ \pm}$-wave gap against disorder, and increasing the overlap between the states reduces the overall robustness. For TRSB disorder, the overlap with the conventional $s$-wave state is detrimental, and increasing the overlap with the unconventional $s$ - wave state by increasing the spin-orbit coupling increases the robustness.

The results of this calculation are presented in Fig. 7. for TRS and TRSB disorder, under the assumption that there is pairing only in the momentum-dependent channel.

\section{DISCUSSION}

The existence of unconventional $s$-wave pairing states in systems with additional internal degrees of freedom has significant consequences for the robustness of superconductivity against disorder. The general framework we have presented provides a straightforward, analytically tractable method to predict the robustness of a particular superconducting state in a given system, and also easily accounts for known results in systems of interest [18, 19, 22, 25]. The superconducting fitness is of critical importance in determining the robustness, as evidenced clearly in the robustness of the $B_{1 g}$ gap in the iron-pnictide model (see Fig. 2), which has a nearly per- 


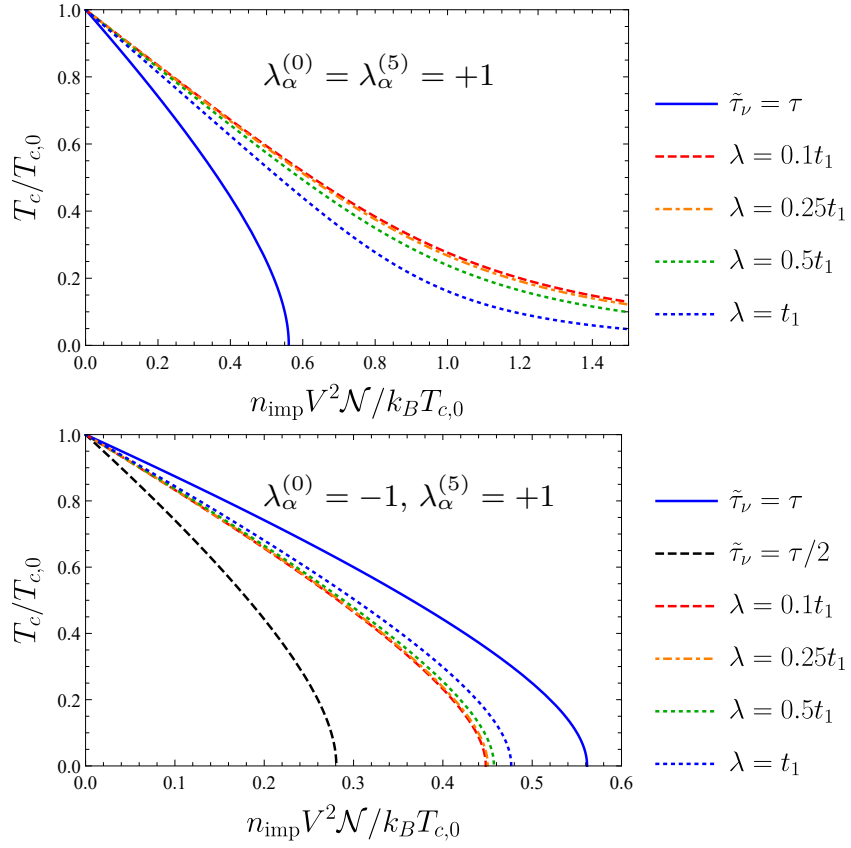

Figure 7. Robustness of the momentum-dependent $s_{ \pm}$-wave $A_{1 g}$ gap against against TRS (top) and TRSB (bottom) disorder, in the two-orbital model for iron oxypnictides with various spin-orbit coupling strengths. The influence of $\lambda_{\alpha}^{(5)}$, the factor for the unconventional $s$-wave state has only a minor influence on the robustness and so we consider only $\lambda_{\alpha}^{(5)}=+1$ here.

fect fitness on one band at the Fermi surface.

Interestingly, the fitness of the superconducting gap with respect to the disorder potential plays perhaps an even more significant role. In our framework, the parameter $\lambda_{\alpha}= \pm 1$ encapsulates the fitness with respect to disorder, and determines whether the spinorbital texture acts to enhance (if $\lambda_{\alpha}=+1$, which requires $\tilde{V}_{\alpha} \tilde{\Delta}_{\nu}-\tilde{\Delta}_{\nu} \tilde{V}_{\alpha}^{T}=0$ ) or reduce (if $\lambda_{\alpha}=-1$, $\tilde{V}_{\alpha} \tilde{\Delta}_{\nu}-\tilde{\Delta}_{\nu} \tilde{V}_{\alpha}^{T} \neq 0$ ) the robustness. This is consistent with Anderson's theorem: for the conventional $s$-wave singlet state of single-band systems, TRS disorder potentials are perfectly fit and TRSB potentials perfectly unfit. As we have demonstrated, when additional degrees of freedom are present, there will exist TRSB disorder potentials for which a given unconventional $s$-wave state is fit, and as a result the superconductor will be more robust against certain forms of TRSB disorder than TRS disorder potentials. The fitness with respect to the disorder potential is therefore a more versatile definition when considering the influence of disorder, as opposed to whether the disorder preserves or breaks time-reversal symmetry.

The important role played by the superconducting fitness in determining the robustness against scalar disorder can be understood via a canonical transformation, $c_{\boldsymbol{k}} \rightarrow \exp \left(i \frac{\pi}{4} \tilde{\Delta}_{\nu} U_{T}^{\dagger}\right) c_{\boldsymbol{k}}$, that maps the unconventional $s$-wave state to a conventional $s$-wave state
9, 19, 25. Under such a transformation, components of the Hamiltonian for which the gap is perfectly fit (i.e. $\varepsilon_{\boldsymbol{k}, i} \gamma^{i} \tilde{\Delta}_{\nu}-\tilde{\Delta}_{\nu}\left[\varepsilon_{\boldsymbol{k}, i} \gamma^{i}\right]^{T}=0$ ) are mapped onto TRS terms, whereas the unfit components are transformed into TRSB terms. The superconductivity is therefore robust against the former terms, but is destabilized by the latter. Similarly, this transformation also maps disorder potentials with $\lambda_{\alpha}=1$ onto TRS disorder potentials, whereas the disorder potentials with $\lambda_{\alpha}=-1$ are mapped onto TRSB potentials. The pairing consequently has enhanced robustness against disorder potentials with $\lambda_{\alpha}=+1$, but the mapping of the unfit elements of the Hamiltonian to TRSB terms violates Anderson's theorem, preventing perfect robustness. On the other hand, the unfit terms of the Hamiltonian reduce the magnitude of the effective scattering rate due to the disorder potentials with $\lambda_{\alpha}=-1$ relative to the value for a conventional $s$-wave, and thereby lead to an enhanced robustness.

We have additionally demonstrated the significant role played by the number of bands that cross the Fermi level. As a clear example, consider the unconventional $s$-wave $A_{1 g}$ pairing state in the two models we consider: in the Dirac system a single band crosses the Fermi surface and the unconventional state is indistinguishable from the conventional $s$-wave, while for the iron pnictide model the unconventional gap component changes sign between the two bands' Fermi surfaces. In the first case, the general $A_{1 g}$ pairing state is always completely robust against TRS disorder, while in the second the unconventional state is sensitive (to a degree determined by the superconducting fitness) to all disorder as is the unconventional component of a general pairing state.

\section{A. Relationship to other work}

As we have noted previously, our framework readily accounts for, and significantly generalizes, recent results for Dirac superconductors in the presence of trivial and nontrivial disorder potentials [18, 25]. More generally, our results are consistent with recent proposals for generalizations of Anderson's Theorem [20, 22. The generalized Anderson's theorem proposed in Ref. [22] is of particular interest, being completely consistent with our own framework, and demonstrating that our results can be straightforwardly generalized to systems with more than two bands. A key point of difference between our result and the generalized theorem of Ref. [22], is our explicit treatment of the spin and orbital degrees of freedom.

Briefly, we wish to compare our results for Dirac systems with recent findings which are apparently inconsistent with our results. Sato and Asano 24 found an evenorbital-parity spin-singlet $s$-wave state in $\mathrm{Cu}_{x} \mathrm{Bi}_{2} \mathrm{Se}_{3}$ (belonging to the $A_{2 u}$ irrep) to be robust against disorder. In the weak disorder limit our results are consistent, but for strong disorder a gradual exponential suppression is seen in Ref. 24, while our framework predicts that superconductivity is completely suppressed for sufficiently 
strong disorder.

In calculating the anomalous part of the self-energy, we have made use of the self-consistent Born approximation, while the authors in 24 consider only the lowest-order Born approximation. While the distinction between the two is not significant in single-band materials, when considering multi-orbital superconductors the lowest-order approximation fails in the strong disorder limit. The result of Ref. 24 is, predictably, consistent with our own for weak disorder, but the two diverge for larger disorder strengths, with Sato and Asano predicting an exponential suppression of $T_{c}$ whereas we find a vanishing critical temperature for strong disorder.

Andersen et al. 23] recently demonstrated a complete robustness of superconductivity against a fit disorder potential in a $\mathrm{Bi}_{2} \mathrm{Se}_{3}$-based superconductor. As we have noted previously [19, however, this complete robustness additionally relies on an implicit assumption that the superconducting state is perfectly fit. Including this additional assumption brings their conclusion into agreement with our own framework as well as other recent results [18, 22].

\section{CONCLUSIONS}

We have presented a general framework, based on the self-consistent Born-approximation, to consider the ro- bustness of superconductivity against various forms of disorder in systems with additional internal degrees of freedom and have highlighted the important role played by the superconducting fitness. Disorder potentials, $\tilde{V}_{\alpha}$, can generally be classified by the fitness of the superconducting state with respect to the disorder potential. The superconducting fitness with regard to the normal state Hamiltonian then defines the degree to which the state is robust against disorder. The spin-orbital texture, as encapsulated by the superconducting fitness, acts to enhance the robustness against disorder for which the gap is fit, but reduces the robustness against unfit forms of disorder. We have also demonstrated how the robustness is influenced by the presence of multiple Fermi surfaces, where the presence of multiple effective scattering rate can significantly alter the robustness, most noticeably for orbitally-trivial unconventional gaps.

\section{ACKNOWLEDGMENTS}

The authors are thankful to David Dentelski, Jonathan Ruhman, Takumi Sato, Yasuhiro Asano, and Peter Orth for helpful discussions. This work was supported by the Marsden Fund Council from Government funding, managed by Royal Society Te Apārangi.
[1] V. P. Mineev and K. V. Samokhin, Introduction to Unconventional Superconductivity (Gordon and Breach Science Publishers, 1999).

[2] P. W. Anderson, Theory of dirty superconductors, J. Phys. Chem. Solids 11, 26-30 (1959)

[3] X.-L. Qi and S.-C. Zhang, Topological insulators and superconductors, Rev. Mod. Phys. 83, 1057 (2011).

[4] T. Nomoto, K. Hattori, and H. Ikeda, Classification of "multipole" superconductivity in multiorbital systems and its implications, Phys. Rev. B 94, 174513 (2016)

[5] A. M. Black-Schaffer and A. V. Balatsky, Odd-frequency superconducting pairing in multiband superconductors, Phys. Rev. B 88, 104514 (2013)

[6] A. Ramires and M. Sigrist, Identifying detrimental effects for multiorbital superconductivity: Application to $\mathrm{Sr}_{2} \mathrm{RuO}_{4}$, Phys. Rev. B 94, 104501 (2016).

[7] M. Yi, Y. Zhang, Z.-X. Shen, and D. Lu, Role of the orbital degree of freedom in iron-based superconductors, npj Quant Mater 2, 57 (2017).

[8] L. Savary, J. Ruhman, J. W. F. Venderbos, L. Fu, and P. A. Lee, Superconductivity in three-dimensional spin-orbit coupled semimetals, Phys. Rev. B 96, 214514 (2017)

[9] L. Fu and E. Berg, Odd-parity topological superconductors: Theory and application to $\mathrm{Cu}_{x} \mathrm{Bi}_{2} \mathrm{Se}_{3}$, Phys. Rev. Lett. 105, 097001 (2010).

[10] O. Vafek and A. V. Chubukov, Hund interaction, spinorbit coupling, and the mechanism of superconductivity in strongly hole-doped iron pnictides, Phys. Rev. Lett.
118, $087003(2017)$

[11] D. F. Agterberg, T. Shishidou, J. O'Halloran, P. M. R. Brydon, and M. Weinert, Resilient nodeless $d$-wave superconductivity in monolayer FeSe, Phys. Rev. Lett. 119, 267001 (2017)

[12] P. M. R. Brydon, L. Wang, M. Weinert, and D. F. Agterberg, Pairing of $j=3 / 2$ fermions in half-Heusler superconductors, Phys. Rev. Lett. 116, 177001 (2016).

[13] T. Kawakami, T. Okamura, S. Kobayashi, and M. Sato, Topological crystalline materials of $J=3 / 2$ electrons: Antiperovskites, dirac points, and high winding topological superconductivity, Phys. Rev. X 8, 041026 (2018)

[14] D. Möckli and M. Khodas, Robust parity-mixed superconductivity in disordered monolayer transition metal dichalcogenides, Phys. Rev. B 98, 144518 (2018).

[15] R. Oiwa, Y. Yanagi, and H. Kusunose, Theory of superconductivity in hole-doped monolayer $\mathrm{MoS}_{2}$, Phys. Rev. B 98, 064509 (2018)

[16] T. Ong, P. Coleman, and J. Schmalian, Concealed $d$-wave pairs in the $s_{ \pm}$condensate of iron-based superconductors, Proceedings of the National Academy of Sciences 113, 5486 (2016)

[17] E. M. Nica, R. Yu, and Q. Si, Orbital-selective pairing and superconductivity in iron selenides, npj Quant Mater 2, 24 (2017).

[18] K. Michaeli and L. Fu, Spin-orbit locking as a protection mechanism of the odd-parity superconducting state against disorder, Phys. Rev. Lett. 109, 187003 (2012) 
[19] D. C. Cavanagh and P. M. R. Brydon, Robustness of unconventional $s$-wave superconducting states against disorder, Phys. Rev. B 101, 054509 (2020)

[20] M. S. Scheurer, M. Hoyer, and J. Schmalian, Pair breaking in multiorbital superconductors: An application to oxide interfaces, Phys. Rev. B 92, 014518 (2015).

[21] M. S. Scheurer, Mechanism, symmetry and topology of ordered phases in correlated systems, Ph.D. thesis (2016).

[22] E. I. Timmons, S. Teknowijoyo, M. Kończykowski, O. Cavani, M. A. Tanatar, S. Ghimire, K. Cho, Y. Lee, L. Ke, N. H. Jo, S. L. Bud'ko, P. C. Canfield, P. P. Orth, M. S. Scheurer, and R. Prozorov, Electron irradiation effects on superconductivity in $\mathrm{PdTe}_{2}$ : An application of a generalized Anderson theorem, Phys. Rev. Research 2, 023140 (2020)

[23] L. Andersen, A. Ramires, Z. Wang, T. Lorenz, and Y. Ando, Generalized Anderson's theorem for superconductors derived from topological insulators, Science Advances 6, 10.1126/sciadv.aay6502 (2020), https://advances.sciencemag.org/content/6/9/eaay6502.full

[24] T. Sato and Y. Asano, Superconductivity in Cu-doped $\mathrm{Bi}_{2} \mathrm{Se}_{3}$ with potential disorder, Phys. Rev. B 102, 024516 (2020).

[25] D. Dentelski, V. Kozii, and J. Ruhman, Effect of interorbital scattering on superconductivity in doped Dirac semimetals, Phys. Rev. Research 2, 033302 (2020)

[26] S.-K. Yip, Models of superconducting $\mathrm{Cu}: \mathrm{Bi}_{2} \mathrm{Se}_{3}$ : Singleversus two-band description, Phys. Rev. B 87, 104505 (2013).

[27] K. Matano, M. Kriener, K. Segawa, Y. Ando, and G.-q. Zheng, Spin-rotation symmetry breaking in the superconducting state of $\mathrm{Cu}_{x} \mathrm{Bi}_{2} \mathrm{Se}_{3}$, Nature Physics 12, 852 (2016).

[28] S. Yonezawa, K. Tajiri, S. Nakata, Y. Nagai, Z. Wang, K. Segawa, Y. Ando, and Y. Maeno, Thermodynamic evidence for nematic superconductivity in $\mathrm{Cu}_{x} \mathrm{Bi}_{2} \mathrm{Se}_{3}, \mathrm{Na}-$ ture Physics 13, 123 (2016)

[29] R. Tao, Y.-J. Yan, X. Liu, Z.-W. Wang, Y. Ando, Q.-H. Wang, T. Zhang, and D.-L. Feng, Direct visualization of the nematic superconductivity in $\mathrm{Cu}_{x} \mathrm{Bi}_{2} \mathrm{Se}_{3}$, Phys. Rev. X 8, 041024 (2018).

[30] L. Fu, Odd-parity topological superconductor with nematic order: Application to $\mathrm{Cu}_{x} \mathrm{Bi}_{2} \mathrm{Se}_{3}$, Phys. Rev. B 90, 100509(R) (2014)

[31] V. Cvetkovic and O. Vafek, Space group symmetry, spinorbit coupling, and the low-energy effective Hamiltonian for iron-based superconductors, Phys. Rev. B 88, 134510 (2013)

32 I. I. Mazin, D. J. Singh, M. D. Johannes, and M. H. Du, Unconventional superconductivity with a sign reversal in the order parameter of $\mathrm{LaFeAsO}_{1-x} \mathrm{~F}_{x}$, Phys. Rev. Lett. 101, 057003 (2008)

[33] A. V. Chubukov, D. V. Efremov, and I. Eremin, Magnetism, superconductivity, and pairing symmetry in ironbased superconductors, Phys. Rev. B 78, 134512 (2008)

[34] A. V. Chubukov, Pairing mechanism in Fe-based superconductors, Annual Review of Condensed Matter Physics 3, 57 (2012), https://doi.org/10.1146/annurevconmatphys-020911-125055
[35] J. Paglione and R. L. Greene, High-temperature superconductivity in iron-based materials, Nature Physics 6, 645-658 (2010).

[36] S. Onari and H. Kontani, Violation of Anderson's theorem for the sign-reversing $s$-wave state of iron-pnictide superconductors, Phys. Rev. Lett. 103, 177001 (2009).

[37] D. V. Efremov, M. M. Korshunov, O. V. Dolgov, A. A. Golubov, and P. J. Hirschfeld, Disorder-induced transition between $s_{ \pm}$and $s_{++}$states in two-band superconductors, Phys. Rev. B 84, 180512(R) (2011)

[38] D. V. Efremov, A. A. Golubov, and O. V. Dolgov, Manifestations of impurity-induced $s^{ \pm} \rightarrow s^{++}$transition: Multiband model for dynamical response functions, $\mathrm{New}$ Journal of Physics 15, 013002 (2013)

39] Y. Yamakawa, S. Onari, and H. Kontani, Effect of realistic finite-size impurities on $T_{c}$ in Fe-based superconductors based on the five-orbital tight-binding model, Phys. Rev. B 87, 195121 (2013)

[40] Y. Wang, A. Kreisel, P. J. Hirschfeld, and V. Mishra, Using controlled disorder to distinguish $s_{ \pm}$and $s_{++}$gap structure in Fe-based superconductors, Phys. Rev. B 87, 094504 (2013)

[41] V. Stanev and A. E. Koshelev, Complex state induced by impurities in multiband superconductors, Phys. Rev. B 89, 100505(R) (2014)

[42] M. Hoyer, M. S. Scheurer, S. V. Syzranov, and J. Schmalian, Pair breaking due to orbital magnetism in iron-based superconductors, Phys. Rev. B 91, 054501 $(2015)$

[43] M. Daghofer, A. Nicholson, A. Moreo, and E. Dagotto, Three orbital model for the iron-based superconductors, Phys. Rev. B 81, 014511 (2010)

[44] S. Raghu, X.-L. Qi, C.-X. Liu, D. J. Scalapino, and S.-C. Zhang, Minimal two-band model of the superconducting iron oxypnictides, Phys. Rev. B 77, 220503(R) (2008)

[45] A. Ramires, D. F. Agterberg, and M. Sigrist, Tailoring $T_{c}$ by symmetry principles: The concept of superconducting fitness, Phys. Rev. B 98, 024501 (2018)

[46] P. M. R. Brydon, D. F. Agterberg, H. Menke, and C. Timm, Bogoliubov Fermi surfaces: General theory, magnetic order, and topology, Phys. Rev. B 98, 224509 (2018).

[47] C.-X. Liu, X.-L. Qi, H. J. Zhang, X. Dai, Z. Fang, and S.C. Zhang, Model Hamiltonian for topological insulators, Phys. Rev. B 82, 045122 (2010)

[48] K. Kuroki, S. Onari, R. Arita, H. Usui, Y. Tanaka, H. Kontani, and H. Aoki, Unconventional pairing originating from the disconnected Fermi surfaces of superconducting $\mathrm{LaFeAsO}_{1-x} \mathrm{~F}_{x}$, Phys. Rev. Lett. 101, 087004 (2008)

[49] H. Eschrig and K. Koepernik, Tight-binding models for the iron-based superconductors, Phys. Rev. B 80, 104503 (2009).

[50] S. Graser, A. F. Kemper, T. A. Maier, H.-P. Cheng, P. J. Hirschfeld, and D. J. Scalapino, Spin fluctuations and superconductivity in a three-dimensional tight-binding model for $\mathrm{BaFe}_{2} \mathrm{As}_{2}$, Phys. Rev. B 81, 214503 (2010).

[51] V. P. Mineev and K. V. Samokhin, Effects of impurities on superconductivity in noncentrosymmetric compounds, Phys. Rev. B 75, 184529 (2007) 